\title{
An approximate approach to the exponential utility indifference valuation
}

\author{
Takuji Arai \\ Faculty of Economics, Keio University, 2-15-45 Mita, Minato-ku, \\ Tokyo, 108-8345, Japan (e-mail: arai@econ.keio.ac.jp)
}

\begin{abstract}
We propose, in this paper, a new valuation method for a contingent claim, which approximates to the exponential utility indifference valuation. In particular, we treat both ask and bid valuations. In the definition of the exponential utility indifference valuation, we require a strong condition related to the underlying contingent claim. The new valuation in this paper succeeds in reducing this condition by using a kind of power functions instead of the exponential function. Furthermore, we shall investigate some basic properties and an asymptotic behavior of our new valuation.
\end{abstract}

Keywords: Incomplete markets, Indifference value, $p$-optimal martingale measure, Reverse Hölder inequality.

\section{Introduction}

Our aim of this paper is to obtain an approximate approach to the exponential utility indifference valuation (EUIV, for short) by using a kind of power functions.

In mathematical finance, the problem of valuation for a contingent claim in an incomplete market is very important. Recently, many researchers have studied the utility indifference valuation method, of which the definition is given by as follows: We start with an incomplete market with the maturity $T>0$, whose asset fluctuation is described by a semimartingale $X$. Moreover, we consider an investor having initial capital $x_{t}$ at time $t$, and who intends to sell a contingent claim $B$. Let $U$ be his/her utility function. In other words, $U$ is an $\mathbf{R}$-valued continuous increasing concave function defined on $\mathbf{R}$. We define an adapted process $C_{t}(B)$ by

$$
\begin{aligned}
& \operatorname{esssup}_{\vartheta \in \Theta} E\left[U\left(x_{t}+G_{t, T}(\vartheta)\right) \mid \mathcal{F}_{t}\right] \\
& \quad=\operatorname{essup}_{\vartheta \in \Theta} E\left[U\left(x_{t}+C_{t}(B)+G_{t, T}(\vartheta)-B\right) \mid \mathcal{F}_{t}\right],
\end{aligned}
$$


where $G_{t, T}(\vartheta):=\int_{t}^{T} \vartheta_{s} d X_{s}$ and $\Theta$ a suitable set of predictable processes, represents the set of all self-financing strategies. Then, we call $C_{t}(B)$ the utility indifference valuation, which is one of candidates for the asking price of the contingent claim $B$. In addition, the valuation $C_{t}(B)$ strongly depends on the preference of the investor who intends to sell $B$. The left hand side of (1.1) is the expected utility maximization problem when he/she does not sell the contingent claim $B$. On the other hand, the right hand side is the case where he/she sells $B$ for the price $C_{t}(B)$ at time $t$ and agrees to pay $B$ at the maturity $T$. In particular, there has been much literature on the exponential utility case, that is, the case where $U$ is given by $U(x)=-e^{-\alpha x}$, for $\alpha>0$. See Becherer (2004), Frittelli (2000), Rouge and El Karoui (2000), Musiela and Zariphopoulou (2004a, 2004b), Young (2004), and so on. Besides, Mania and Schweizer (2005) (MS, for short) have provided the dynamics for the case where the asset price process is given by a continuous semimartingale. Remark that we call $C_{t}(B)$ the exponential utility indifference valuation (EUIV), if $U$ is the exponential utility function.

On the other hand, when we define the EUIV, we need to assume the following strong condition with respect to the underlying contingent claim:

$$
E\left[e^{\alpha B}\right]<\infty .
$$

For example, in the case where $B$ is a European call option and $X$ is given by a geometric Brownian motion, (1.2) does not hold, because, roughly speaking, the distribution of $B$ is near to one of $e^{Y}$, where $Y$ is a normal random variable. Hence, models satisfying the condition (1.2) do not include some typical important ones as the above example. At this, we try to reduce the condition (1.2) to, for a sufficient large $n \in \mathbf{N}$,

$$
E\left[B^{n}\right]<\infty
$$

equivalently $E\left[e^{n Y}\right]<\infty$. Now, we recall the definition of " $e$ " as follows:

$$
e^{x}=\lim _{n \rightarrow \infty}\left(1+\frac{x}{n}\right)^{n}
$$

then, for any sufficient large $n$, we can say that $\left(1+\frac{x}{n}\right)^{n}$ is near to $e^{x}$. If we denote, for a sufficient large number $n$,

$$
U(x)=-\left(1+\frac{\alpha x}{n}\right)^{-n} \text { or }-\left(1-\frac{\alpha x}{n}\right)^{n},
$$

then we can approximate the EUIV under the condition (1.3). Remark that this function $U$ is not a utility function exactly, since not concave. Although, for $x<$ $n / \alpha, U$ is concave, so that we can say that $U$ is almost concave. Instead of the exponential utility, if we adopt the function $U$ as the underlying utility function, then we may obtain an approximate approach to the EUIV. On the other hand, it is difficult for us to treat $U$ directly. Therefore, we try to decompose the 
value $x_{t}+C_{t}(B)+G_{t, T}(\vartheta)-B$ into the $\mathcal{F}_{t}$-measurable part $x_{t}+C_{t}(B)$, the gain process part $G_{t, T}(\vartheta)$ and the contingent claim part $B$. Thus, instead of $U$, we consider, for $\alpha>0$ and $n \in \mathbf{N}$,

$$
U_{\alpha, n}(x, y, z):=-\left(1+\frac{\alpha x}{n}\right)^{-n}\left(1-\frac{\alpha y}{n}\right)^{n+1}\left(1+\frac{\alpha z}{n}\right)^{n} .
$$

Note that, if $n$ is sufficient large, then $U_{\alpha, n}(x, y, z)$ is very near to $-\left(1+\frac{\alpha}{n}(x+y-z)\right)^{-n}$ or $-\left(1-\frac{\alpha}{n}(x+y-z)\right)^{n}$. On the other hand, if we denote

$$
U_{\alpha, \exp }(x, y, z):=-\exp (-\alpha(x+y-z)),
$$

then the EUIV, denoted by $C_{t}^{\alpha, \exp }(B)$, satisfies the following:

$$
\begin{aligned}
& \operatorname{esssup}_{\vartheta \in \Theta} E\left[U_{\alpha, \exp }\left(x_{t}, G_{t, T}(\vartheta), 0\right) \mid \mathcal{F}_{t}\right] \\
& \quad=\operatorname{esssup}_{\vartheta \in \Theta} E\left[U_{\alpha, \exp }\left(x_{t}+C_{t}^{\alpha, \exp }(B), G_{t, T}(\vartheta), B\right) \mid \mathcal{F}_{t}\right] .
\end{aligned}
$$

Remark that $C_{t}^{\alpha, \exp }(B)$ does not depend on the initial capital $x_{t}$. Thus, by the same way as the EUIV, we define an adapted process $C_{t}^{\alpha, n}(B)$ as a process satisfying

$$
\begin{aligned}
& \operatorname{esssup}_{\vartheta \in \Theta} E\left[U_{\alpha, n}\left(x_{t}, G_{t, T}(\vartheta), 0\right) \mid \mathcal{F}_{t}\right] \\
& \quad=\operatorname{esssup}_{\vartheta \in \Theta} E\left[U_{\alpha, n}\left(x_{t}+C_{t}^{\alpha, n}(B), G_{t, T}(\vartheta), B\right) \mid \mathcal{F}_{t}\right] .
\end{aligned}
$$

This process $C_{t}^{\alpha, n}(B)$ may be a strong candidate of approximations to the EUIV. Hence, we shall investigate some properties of $C_{t}^{\alpha, n}(B)$ in this paper. Remark that $C_{t}^{\alpha, n}(B)$ depends on $x_{t}$. Henceforth, we fix $x_{t}=0$.

The structure of this paper is as follows: In Section 2, we state the standing assumptions and the exact definition of our new valuation $C_{t}^{\alpha, n}(B)$. In particular, we need the closedness of the set of all self-financing strategies in the $\mathcal{L}^{n+1}$ sense. This closedness is close related to the $1+\frac{1}{n}$-optimal martingale measure. Thus, some standing assumptions are concerned in it. Moreover, remark that it is close related to the projection of "1" onto a suitable space of the stochastic integrations. In addition, we introduce, in Section 3, an example satisfying the all standing assumptions.

In order to make sure that our new valuation is useful as an approximate approach to the EUIV, we investigate its basic properties and the asymptotic behavior as $n$ tends to 0 . In Section 4, we prove that our new valuation has same basic properties as the EUIV approximately. In particular, we show that there exists a duality relationship between a portfolio optimization problem related to our new valuation and an optimization problem among equivalent martingale measures, which is related to the $1+\frac{1}{n}$-optimal martingale measure. Furthermore, we assert in Section 5 that $C_{t}^{\alpha, n}(B)$ converges to the EUIV as $n$ tends to $\infty$ in probability. To see this, it is worth while to notice that the $p$-optimal martingale measure converges to the minimal martingale measure as 
$p$ tends to 1, which has been proved by Grandits and Rheinländer (2002) (GR, for short).

On the other hand, we can say that the definition of the utility indifference valuation is an ask-pricing method, which is one from a seller's view. Thus, in Section 6, we extend our new valuation to one from a buyer's view. That is, we treat a bid-pricing method. In addition, we introduce a duality relation as in Section 4, and investigate some basic properties of the valuation from a buyer's view.

\section{Preliminaries}

In this section, we introduce the three standing assumptions and some notations. Moreover, we formulate the exact definition of our new valuation $C_{t}^{\alpha, n}(B)$ under the standing assumptions. In other words, we give the definition of the set of all self-financing strategies.

Throughout this paper, we consider an incomplete financial market composed of one riskless asset whose price is "1" at all time, and $d$ risky assets described by an $\mathbf{R}^{d}$-valued continuous semimartingale $X$. Suppose that the maturity is $T>0$. Let $\left(\Omega, \mathcal{F}, P ; \mathbf{F}=\left\{\mathcal{F}_{t}\right\}_{t \in[0, T]}\right)$ be a completed filtered probability space with a right-continuous filtration $\mathbf{F}$ such that $\mathcal{F}_{0}$ is trivial and contains all null sets of $\mathcal{F}$, and $\mathcal{F}_{T}=\mathcal{F}$. Furthermore, in this paper, we treat a suitable set of $\mathbf{R}^{d}$-valued predictable $X$-integrable processes $\vartheta$ as the set of all self-financing strategies, denoted by $\Theta$. Let $B$ be an $\mathcal{F}_{T}$-measurable random variable. Throughout this paper, we regard $B$ as a contingent claim, that is, a pay-off at the maturity $T$. We fix a positive real number $\alpha$ and a large odd number $n$. To simplify notations, we restrict $n$ within odd numbers. For all unexplained notations, we refer to Dellacherie and Meyer (1982) and GR. Throughout this paper, $C$ denotes a constant in $(0, \infty)$ which may vary from line to line.

Firstly, we give one of the standing assumptions related to the underlying contingent claim $B$.

Assumption 2.1 We assume that $B \geq 0$ and $B \in \mathcal{L}^{n}(P)$.

In the definition of the EUIV, we do not assume the positivity of $B$. However, since the term $\left(1+\frac{\alpha}{n} B\right)^{-1}$ appears in the sequel, we restrict $B$ to positive in this paper.

Next, we prepare some notations in order to introduce the other standing assumptions. Let $P^{0}$ be a probability measure which is equivalent to $P$, and $p>1$.

Definition 2.2 (1) Let $S \leq T$ be a stopping time. We denote by ${ }^{S} \mathcal{V}\left(P^{0}\right)$ the linear subspace of $\mathcal{L}^{\infty}\left(P^{0}\right)$ spanned by the simple stochastic integrals of the form $h^{\operatorname{tr}}\left(X_{T_{2}}-X_{T_{1}}\right)$, where $S \leq T_{1} \leq T_{2} \leq T$ are stopping times such that the stopped process $X^{T_{2}}$ is bounded, and $h$ is a bounded $\mathbf{R}^{d}$-valued $\mathcal{F}_{T_{1}}$-measurable 
random variable. Set $\mathcal{V}\left(P^{0}\right)={ }^{0} \mathcal{V}\left(P^{0}\right)$.

(2) A signed martingale measure under $P^{0}$ is a signed measure $Q \ll P^{0}$ with $E_{P^{0}}\left[\frac{d Q}{d P^{0}}\right]=1$ and $E_{P^{0}}\left[\frac{d Q}{d P^{0}} f\right]=0$ for all $f \in \mathcal{V}\left(P_{0}\right)$.

(3) $\mathcal{M}^{s}\left(P^{0}\right)$ is the space of all signed martingale measures under $P^{0}$, and $\mathcal{M}^{e}\left(P^{0}\right)$ is the subset of $\mathcal{M}^{s}\left(P^{0}\right)$ consisting of probability measures being equivalent to $P^{0}$. Moreover, we set $\mathcal{M}_{p}^{x}\left(P^{0}\right):=\mathcal{M}^{x}\left(P^{0}\right) \cap \mathcal{L}^{p}\left(P^{0}\right)$ for $x \in\{e, s\}$.

(4) The $p$-optimal martingale measure with respect to $P^{0}$ is defined as the element of $\mathcal{M}_{p}^{s}\left(P^{0}\right)$ which minimizes $\mathcal{L}^{p}\left(P^{0}\right)$-norm.

(5) Let $Y$ be a uniformly integrable $P^{0}$-martingale with $Y_{0}=1$ and $Y_{T}>0$. We say that $Y$ satisfies the reverse Hölder inequality $\mathcal{R}_{p}\left(P^{0}\right)$, if there is a constant $C$ such that for every stopping time $S \leq T$, we have

$$
E_{P^{0}}\left[\left(\frac{Y_{T}}{Y_{S}}\right)^{p} \mid \mathcal{F}_{S}\right] \leq C
$$

The $1+\frac{1}{n}$-optimal martingale measure will play an important role, so that the following assumption is essential.

Assumption 2.3 We assume that the $1+\frac{1}{n}$-optimal martingale measure $Q^{(n)}$ exists in $\mathcal{M}_{1+\frac{1}{n}}^{e}(P)$, and its density process $Z^{(n)}$ satisfies the reverse Hölder inequality $\mathcal{R}_{1+\frac{1}{n}}(P)$.

Since $X$ is a continuous semimartingale, it is special under $P$, and its canonical decomposition is given by $X=X_{0}+M+A$ with $M$ a local martingale, $A$ a predictable process, and $M_{0}=A_{0}=0$. Moreover, if $P^{0}$ is equivalent to $P$, then $X$ is also a special semimartingale under $P^{0}$. Let us denote its canonical decomposition under $P^{0}$ as follows:

$$
X=X_{0}+M^{0}+A^{0} .
$$

Definition 2.4 (1) We denote by ${ }^{S} \mathcal{K}_{p}\left(P^{0}\right)$ the closure in $\mathcal{L}^{p}\left(P^{0}\right)$ of ${ }^{S} \mathcal{V}\left(P^{0}\right)$ for a stopping time $S \leq T$. In particular, let $\mathcal{K}_{p}\left(P^{0}\right):={ }^{0} \mathcal{K}_{p}\left(P^{0}\right)$.

(2) Let $L^{p}\left(M^{0}\right)$ be the space of all $\mathbf{R}^{d}$-valued predictable processes $\vartheta$ such that

$$
\|\vartheta\|_{L^{p}\left(M^{0}\right)}:=E_{P^{0}}^{1 / p}\left[\left(\int \vartheta^{\operatorname{tr}} d\left[M^{0}\right] \vartheta\right)_{T}^{p / 2}\right]<\infty .
$$

(3) Let $L^{p}\left(A^{0}\right)$ be the space of all $\mathbf{R}^{d}$-valued predictable processes $\vartheta$ such that

$$
\|\vartheta\|_{L^{p}\left(A^{0}\right)}:=E_{P^{0}}^{1 / p}\left[\left(\int\left|\vartheta^{\operatorname{tr}} d A^{0}\right|\right)_{T}^{p}\right]<\infty .
$$

(4) A positive process $Y$ satisfies $\mathcal{R}_{L \log L}\left(P^{0}\right)$ if there exists a constant $C>0$ such that

$$
\sup _{S}\left\|E_{P^{0}}\left[\frac{Y_{T}}{Y_{S}} \log ^{+} \frac{Y_{T}}{Y_{S}} \mid \mathcal{F}_{S}\right]\right\|_{\infty} \leq C
$$


where the supremum is taken over all stopping times $S \leq T$.

We define

$$
\Theta^{n+1}\left(P^{0}\right):=L^{n+1}\left(A^{0}\right) \cap L^{n+1}\left(M^{0}\right)
$$

and

$$
G_{t, T}(\Theta):=\left\{\int_{t}^{T} \vartheta_{s} d X_{s} \mid \vartheta \in \Theta\right\},
$$

for a suitable set $\Theta$ of $\mathbf{R}^{d}$-valued $X$-integrable predictable processes. In particular, we denote $G_{T}(\Theta):=G_{0, T}(\Theta)$. Remark that we can rearrange the definition of $\Theta^{n+1}\left(P^{0}\right)$ as

$$
\Theta^{n+1}\left(P^{0}\right):=\left\{\vartheta \mid G(\vartheta) \in \mathcal{S}^{n+1}\left(P^{0}\right)\right\} .
$$

By Theorem 4.1 of Grandits and Krawczyk (1998), $G_{T}\left(\Theta^{n+1}(P)\right)$ is $\mathcal{L}^{n+1}(P)$ closed under Assumption 2.3. Proposition 4.7 of GR yields that there exists the minimal entropy martingale measure the density process of which satisfies $\mathcal{R}_{L \log L}(P)$. In addition, Lemma 2.1 of GR implies

$$
G_{t, T}\left(\Theta^{n+1}(P)\right)={ }^{t} \mathcal{K}_{n+1}(P) .
$$

Moreover, since $n$ is odd, Propositions 4.2 and 4.4 of GR imply, by passing to a version if necessary,

$$
Z_{t, T}^{(n)}:=Z_{T}^{(n)} / Z_{t}^{(n)}=C_{t}^{(n)}\left(1+\frac{{ }^{t} f_{T}^{(n)}}{n}\right)^{n},
$$

where

$$
Z_{t}^{(n)}:=E\left[\frac{d Q^{(n)}}{d P} \mid \mathcal{F}_{t}\right]
$$

$C_{t}^{(n)}$ is an $\mathcal{F}_{t^{-}}$-measurable positive random variable, and ${ }^{t} f_{T}^{(n)} \in{ }^{t} \mathcal{K}_{n+1}(P)$. In particular, $-{ }^{t} f_{T}^{(n)} / n$ is the projection of " 1 " onto ${ }^{t} \mathcal{K}_{n+1}(P)$ in $\mathcal{L}^{n+1}(P)$.

Thirdly, we define a probability measure $P^{n, B}$ as

$$
\frac{d P^{n, B}}{d P}:=C^{n, B}\left(1+\frac{\alpha}{n} B\right)^{n},
$$

where $C^{n, B} \in \mathbf{R}_{+}$. Furthermore, we denote

$$
Z_{t, T}^{n, B}:=\frac{Z_{T}^{n, B}}{Z_{t}^{n, B}}=C_{t}^{n, B}\left(1+\frac{\alpha}{n} B\right)^{n} \quad \text { and } \quad Z_{t}^{n, B}:=E\left[\frac{d P^{n, B}}{d P} \mid \mathcal{F}_{t}\right],
$$

where $C_{t}^{n, B}$ is an $\mathcal{F}_{t}$-measurable positive random variable. Remark that $X$ is also a semimartingale under $P^{n, B}$. 
Assumption 2.5 We assume that the $1+\frac{1}{n}$-optimal martingale measure $Q^{(n), B}$ with respect to $P^{n, B}$ exists in $\mathcal{M}_{1+\frac{1}{n}}^{e}\left(P^{n, B}\right)$, and its density process $Z^{(n), B}$ with respect to $P^{n, B}$ satisfies $\mathcal{R}_{1+\frac{1}{n}}\left(P^{n, B}\right)$, where

$$
Z_{t}^{(n), B}:=E_{P^{n, B}}\left[\frac{d Q^{(n), B}}{d P^{n, B}} \mid \mathcal{F}_{t}\right] .
$$

We have

$$
Z_{t, T}^{(n), B}:=Z_{T}^{(n), B} / Z_{t}^{(n), B}=C_{t}^{(n), B}\left(1+\frac{{ }^{t} f_{T}^{(n), B}}{n}\right)^{n},
$$

where $C_{t}^{(n), B}$ is an $\mathcal{F}_{t}$-measurable positive random variable, and ${ }^{t} f_{T}^{(n), B} \in$ ${ }^{t} \mathcal{K}_{n+1}\left(P^{n, B}\right)$. In particular, $-{ }^{t} f_{T}^{(n), B} / n$ is the projection of " 1 " onto ${ }^{t} \mathcal{K}_{n+1}\left(P^{n, B}\right)$ in $\mathcal{L}^{n+1}\left(P^{n, B}\right)$.

In order to define the process $C_{t}^{\alpha, n}(B)$ exactly, we have to determine the set of all self-financing strategies. Note that we have, for an $\mathbf{R}^{d}$-valued predictable process $\vartheta$

$E\left[\left(1-\frac{\alpha}{n} G_{t, T}(\vartheta)\right)^{n+1}\left(1+\frac{\alpha}{n} B\right)^{n} \mid \mathcal{F}_{t}\right]=\frac{1}{C_{t}^{n, B}} E_{P^{n, B}}\left[\left(1-\frac{\alpha}{n} G_{t, T}(\vartheta)\right)^{n+1} \mid \mathcal{F}_{t}\right]$.

Moreover, by the same sort of argument as the above, $G_{T}\left(\Theta^{n+1}\left(P^{n, B}\right)\right)$ is $\mathcal{L}^{n+1}\left(P^{n, B}\right)$-closed under Assumption 2.5. Thus, there exists a solution to the following minimization problem:

$$
\max _{\vartheta \in \Theta^{n+1}\left(P^{n, B}\right)} E\left[\left(1-\frac{\alpha}{n} G_{t, T}(\vartheta)\right)^{n+1}\left(1+\frac{\alpha}{n} B\right)^{n} \mid \mathcal{F}_{t}\right] .
$$

Hence, $\Theta^{n+1}\left(P^{n, B}\right)$ should be the set of all self-financing strategies. Now, we define

$$
\widetilde{\Theta}^{n+1}\left(P^{n, B}\right):=\left\{\vartheta \mid G(\vartheta) \text { is a } Q^{(n), B} \text {-martingale and } G_{T}(\vartheta) \in \mathcal{L}^{n+1}\left(P^{n, B}\right)\right\} .
$$

In addition, we need one more preparation.

Definition 2.6 A $P^{0}$-martingale $Y$ is in $\operatorname{bmo}_{p}\left(P^{0}\right)$ if there exists a constant $C$ such that, for any $t \in[0, T]$,

$$
E_{P^{0}}\left[\left([Y]_{T}-[Y]_{t}\right)^{p / 2} \mid \mathcal{F}_{t}\right] \leq C .
$$

Then, we have the following relationship under Assumptions 2.1, 2.3 and 2.5:

Lemma 2.7 We have $\Theta^{n+1}\left(P^{n, B}\right)=\widetilde{\Theta}^{n+1}\left(P^{n, B}\right)$. 
Proof. Firstly, we prove that, for any $\vartheta \in \Theta^{n+1}\left(P^{n, B}\right), G(\vartheta)$ is a local $Q^{(n), B}$-martingale. Let the canonical decomposition of $X$ under $P^{n, B}$ be given by

$$
X=X_{0}+M^{n, B}+A^{n, B} .
$$

By Lemma 4.6 of Grandits and Krawczyk (1998), the density process $Z^{(n), B}$ of $Q^{(n), B}$ with respect to $P^{n, B}$ is denoted by

$$
Z^{(n), B}=\mathcal{E}\left(N^{(n), B}\right),
$$

where $N^{(n), B}$ is a $P^{n, B}$-martingale and in $\mathrm{bmo}_{1+\frac{1}{n}}\left(P^{n, B}\right)$. On the other hand, since $G(\vartheta) \in \mathcal{S}^{n+1}\left(P^{n, B}\right),\left[\int \vartheta d M^{n, B}\right]$ is in $\mathcal{L}^{(n+1) / 2}\left(P^{n, B}\right)$. Thus, we have

$$
\begin{aligned}
& \left.E_{P^{n, B}}\left[\mid\left[\int \vartheta d M^{n, B}, N^{(n), B}\right]_{T}\right\rfloor\right] \\
& \leq\left\|\left[\int \vartheta d M^{n, B}\right]_{T}^{\frac{1}{2}}\right\|_{\mathcal{L}^{n+1}\left(P^{n, B}\right)}\left\|\left[N^{(n), B}\right]_{T}^{\frac{1}{2}}\right\|_{\mathcal{L}^{1+\frac{1}{n}}\left(P^{n, B}\right)} \\
& \quad<\infty
\end{aligned}
$$

that is, $\left[\int \vartheta d M^{n, B}, N^{(n), B}\right]$ is $P^{n, B}$-integrable. Since the product $Z^{(n), B} X$ is a local $P^{n, B}$-martingale, we obtain $A^{n, B}=-\left\langle N^{(n), B}, M^{n, B}\right\rangle$. Thus, we have, for any $\vartheta \in \Theta^{n+1}\left(P^{n, B}\right)$,

$$
\int \vartheta^{\operatorname{tr}} d A^{n, B}=-\int \vartheta^{\operatorname{tr}} d\left\langle N^{(n), B}, M^{n, B}\right\rangle .
$$

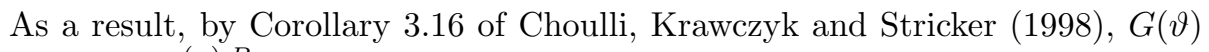
is a local $Q^{(n), B}$-martingale.

In addition, by Theorem V.2 of Protter (1990), there exists a $C>0$ such that

$$
\left\|G_{T}(\vartheta)\right\|_{\mathcal{L}^{1+\frac{1}{n}\left(P^{n, B}\right)}} \leq\left\|\sup _{0 \leq t \leq T} G_{t}(\vartheta)\right\|_{\mathcal{L}^{1+\frac{1}{n}\left(P^{n, B}\right)}} \leq C\|G(\vartheta)\|_{\mathcal{S}^{n+1}\left(P^{n, B}\right)}<\infty
$$

Since $G(\vartheta)$ is a local $Q^{(n), B}$-martingale, $G(\vartheta)$ is a $Q^{(n), B}$-martingale. Thus, we obtain " $\subseteq$ ".

Suppose that $\vartheta \in \widetilde{\Theta}^{n+1}\left(P^{n, B}\right)$. By Theorem 4.12 of Choulli, Krawczyk and Stricker (1999), there exists a constant $C>0$ such that

$$
\|G(\vartheta)\|_{\mathcal{S}^{n+1}\left(P^{n, B}\right)} \leq C\left\|G_{T}(\vartheta)\right\|_{\mathcal{L}^{n+1}\left(P^{n, B}\right)}<\infty .
$$

Hence, $\vartheta \in \Theta^{n+1}\left(P^{n, B}\right)$, from which " $\supseteq$ " holds.

By Lemma 2.7, we can consider that $\widetilde{\Theta}^{n+1}\left(P^{n, B}\right)$ is appropriate as the set of all self-financing strategies. Now, we denote

$$
\Theta_{B}^{(n)}:=\Theta^{n+1}\left(P^{n, B}\right)=\widetilde{\Theta}^{n+1}\left(P^{n, B}\right) .
$$


Again, we define the process $C_{t}^{\alpha, n}(B)$ as follows:

$$
\begin{aligned}
& \operatorname{esssup}_{\vartheta \in \Theta_{0}^{(n)}} E\left[U_{\alpha, n}\left(0, G_{t, T}(\vartheta), 0\right) \mid \mathcal{F}_{t}\right] \\
& \quad=\operatorname{esssup}_{\vartheta \in \Theta_{B}^{(n)}} E\left[U_{\alpha, n}\left(C_{t}^{\alpha, n}(B), G_{t, T}(\vartheta), B\right) \mid \mathcal{F}_{t}\right],
\end{aligned}
$$

where $U_{\alpha, n}$ is given by $U_{\alpha, n}(x, y, z)=-\left(1+\frac{\alpha x}{n}\right)^{-n}\left(1-\frac{\alpha y}{n}\right)^{n+1}\left(1+\frac{\alpha z}{n}\right)^{n}$.

On the other hand, as for the exponential utility case, we define

$$
\Theta^{\exp }:=\left\{\vartheta \in L(X) \mid G(\vartheta) \text { is a } Q \text {-martingale for all } Q \in \mathcal{M}_{\mathrm{ent}}^{e}(P)\right\},
$$

and

$$
\mathcal{M}_{\mathrm{ent}}^{e}(P):=\left\{Q \in \mathcal{M}^{e}(P) \mid H(Q \mid P)<\infty\right\},
$$

where $H(Q \mid P)=E\left[\frac{d Q}{d P} \log \frac{d Q}{d P}\right]$, if $Q \ll P,=\infty$, otherwise. Then, the exponential utility indifference valuation (EUIV) $C_{t}^{\alpha, \exp }(B)$ is defined as an $\mathcal{F}$-adapted process satisfying

$$
\begin{aligned}
& \operatorname{esssup}_{\vartheta \in \Theta^{\exp }} E\left[U_{\alpha, \exp }\left(0, G_{t, T}(\vartheta), 0\right) \mid \mathcal{F}_{t}\right] \\
& \quad=\operatorname{esssup}_{\vartheta \in \Theta^{\exp }} E\left[U_{\alpha, \exp }\left(C_{t}^{\alpha, \exp }(B), G_{t, T}(\vartheta), B\right) \mid \mathcal{F}_{t}\right]
\end{aligned}
$$

where $U_{\alpha, \exp }(x, y, z)=-\exp (-\alpha(x+y-z))$.

\section{$3 \quad$ Examples}

In this section, we introduce an example satisfying Assumptions 2.1, 2.3 and 2.5. Although Assumptions 2.1 and 2.3 are natural comparatively, Assumption 2.5 is artifical one. Then, we have to reveal that models satisfying these assumptions include some important and typical cases.

To be simplicity, we consider only the case where $d=1$. Let $W:=$ $\left(W^{1}, W^{2}, \ldots, W^{l}\right)$ be an $l$-dimensional Brownian motion, where $l \geq 2$. Suppose that the filtration $\mathbf{F}$ is the $P$-augmentation of the filtration generated by $W$, and there exists a predictable process $\lambda$ such that the canonical decomposition of $X$ is given by

$$
X_{t}=X_{0}+M_{t}+A_{t}=X_{0}+M_{t}+\int_{0}^{t} \lambda_{s} d\langle M\rangle_{s} .
$$

In other words, we assume what is called the structure condition (SC) for the process $X$. Moreover, we denote

$$
\widehat{K}_{t}:=\int_{0}^{t} \lambda_{s}^{2} d\langle M\rangle_{s}
$$


which is called the mean-variance trade-off process. Then, we assume that $\widehat{K}$ is bounded. Furthermore, in view of the martingale representation theorem, we can represent $M$ as

$$
M_{t}=\sum_{i=1}^{l} \int_{0}^{t} \sigma_{s}^{i} d W_{s}^{i}
$$

for some $\mathbf{R}^{l}$-valued predictable process $\sigma$. Thus, $X$ is represented as

$$
X_{t}=X_{0}+\sum_{i=1}^{l} \int_{0}^{t} \sigma_{s}^{i} d W_{s}^{i}+\sum_{i=1}^{l} \int_{0}^{t} \lambda_{s}\left(\sigma_{s}^{i}\right)^{2} d s
$$

Now, we assume that $X$ is in $\mathcal{L}^{n}(P)$ and $e^{X}$ is not integrable. For example, the case where $\sigma_{t}=X_{t} \eta_{t}$ and $\lambda_{t}=\zeta_{t} / X_{t}$ for some $\mathbf{R}^{l}$ and $\mathbf{R}$-valued bounded predictable processes $\eta$ and $\zeta$, respectively. In addition, we assume that each $\left|\eta^{i}\right|$ and $|\zeta|$ are positive far away from 0.

Next, suppose that the underlying contingent claim $B$ satisfies Assumption 2.1. For example, the European call option $\left(X_{T}-K\right)^{+}$, where $K$ is its strike price. Since $B \in \mathcal{L}^{n}(P)$, we have, by Corollary 4 of Theorem IV.42 of Protter (1990),

$$
\left(1+\frac{\alpha}{n} B\right)^{n}=E\left[\left(1+\frac{\alpha}{n} B\right)^{n}\right] \mathcal{E}_{T}\left(\sum_{i=1}^{l} \int_{0}^{.} \nu_{s}^{i} d W_{s}^{i}\right),
$$

where $\nu^{i}$ is a predictable process such that $\int_{0}^{T}\left(\nu_{s}^{i}\right)^{2} d s<\infty$. Let $\widehat{P}$ be the minimal martingale measure (see Föllmer and Schweizer (1991)), so that its density process $\widehat{Z}$ is given by

$$
\widehat{Z}_{t}=\mathcal{E}_{t}\left(-\int_{0}^{\cdot} \lambda_{s} d M_{s}\right)=\exp \left(-\int_{0}^{t} \lambda_{s} d M_{s}-\frac{1}{2} \int_{0}^{t} \lambda_{s}^{2} d\langle M\rangle_{s}\right) .
$$

Remark that $\widehat{P}$ is in $\mathcal{M}_{1+\frac{1}{n}}^{e}(P)$, so that Assumption 2.3 is satisfied by Theorem 4.1 of Grandits and Krawczyk (1998). As for $\widehat{Z}$, we have the following representation:

$$
\widehat{Z}=\exp \left(-\sum_{i=1}^{l} \int_{0}^{t} \lambda_{s} \sigma_{s}^{i} d W_{s}^{i}-\frac{1}{2} \sum_{i=1}^{l} \int_{0}^{t}\left(\lambda_{s} \sigma_{s}^{i}\right)^{2} d s\right) .
$$

Hence, if we denote

$$
\widehat{Z}_{t}^{n, B}:=E_{P^{n, B}}\left[\frac{d \widehat{P}}{d P^{n, B}} \mid \mathcal{F}_{t}\right],
$$

then there exists a constant $C>0$ such that

$$
E_{P^{n, B}}\left[\left(\frac{\widehat{Z}_{T}^{n, B}}{\widehat{Z}_{t}^{n, B}}\right)^{1+\frac{1}{n}} \mid \mathcal{F}_{t}\right]
$$




$$
\begin{aligned}
= & E\left[\left(\frac{\widehat{Z}_{T}}{\widehat{Z}_{t}}\right)^{1+\frac{1}{n}}\left(\frac{Z_{T}^{n, B}}{Z_{t}^{n, B}}\right)^{-\frac{1}{n}} \mid \mathcal{F}_{t}\right] \\
= & E\left[\mathcal{E}_{t, T}^{1+\frac{1}{n}}\left(-\int_{0} \lambda_{s} d M_{s}\right) \mathcal{E}_{t, T}^{-\frac{1}{n}}\left(\sum_{i=1}^{l} \int_{0} \nu_{s}^{i} d W_{s}^{i}\right) \mid \mathcal{F}_{t}\right] \\
= & E\left[\exp \left(-\int_{t}^{T}\left(1+\frac{1}{n}\right) \lambda_{s} d M_{s}-\frac{1}{2} \int_{t}^{T}\left(1+\frac{1}{n}\right) \lambda_{s}^{2} d\langle M\rangle_{s}\right)\right. \\
& \left.\times \exp \left(-\frac{1}{n} \sum_{i=1}^{l} \int_{t}^{T} \nu_{s}^{i} d W_{s}^{i}+\frac{1}{2 n} \sum_{i=1}^{l} \int_{t}^{T}\left(\nu_{s}^{i}\right)^{2} d s\right) \mid \mathcal{F}_{t}\right] \\
= & E\left[\mathcal{E}_{t, T}\left(-\sum_{i=1}^{l} \int_{0}^{\cdot}\left(\frac{n+1}{n} \lambda_{s} \sigma_{s}^{i}+\frac{\nu_{s}^{i}}{n}\right)^{i} d W_{s}^{i}\right)\right. \\
& \left.\times \exp \left(\frac{n+1}{2} \sum_{i=1}^{l} \int_{t}^{T}\left(\frac{\lambda_{s} \sigma_{s}^{i}+\nu_{s}^{i}}{n}\right)^{2} d s\right) \mid \mathcal{F}_{t}\right] \\
\leq & C,
\end{aligned}
$$

where $\mathcal{E}_{t, T}:=\mathcal{E}_{T} / \mathcal{E}_{t}$, that is, $\widehat{P}$ satisfies $\mathcal{R}_{1+\frac{1}{n}}\left(P^{n, B}\right)$. Hence, we can conclude that Assumption 2.5 is satisfied.

In summary, the models of which the asset price is expressed by (3.1) satisfy the all standing assumptions under the following conditions:

(1) $X$ is in $\mathcal{L}^{n}(P)$,

(2) the underlying filtration $\mathbf{F}$ is given by the $P$-augmentation of the Brownian motion,

(3) the mean-variance trade-off process is uniformly bounded.

\section{Duality and some properties}

We focus on some basic properties of our new valuation $C_{t}^{\alpha, n}(B)$ in this section. In particular, we are interested whether or not $C_{t}^{\alpha, n}(B)$ satisfies the same basic properties as the EUIV. To see this, we need some preparations.

For an $\mathcal{F}_{t}$-measurable random variable $x_{t}$, we define

$$
V_{t}^{\alpha, n, B}\left(x_{t}\right):=\operatorname{esssup}_{\vartheta \in \Theta_{B}^{(n)}} E\left[U_{\alpha, n}\left(x_{t}, G_{t, T}(\vartheta), B\right) \mid \mathcal{F}_{t}\right] .
$$

Then, we can rewrite the definition of $C_{t}^{\alpha, n}(B)$ as

$$
V_{t}^{\alpha, n, 0}(0)=V_{t}^{\alpha, n, B}\left(C_{t}^{\alpha, n}(B)\right) .
$$

We have

$$
\frac{V_{t}^{\alpha, n, B}(0)}{V_{t}^{\alpha, n, 0}(0)}=\frac{V_{t}^{\alpha, n, B}(0)}{V_{t}^{\alpha, n, B}\left(C_{t}^{\alpha, n}(B)\right)}=\left(1+\frac{\alpha}{n} C_{t}^{\alpha, n}(B)\right)^{n}
$$


namely,

$$
C_{t}^{\alpha, n}(B)=\frac{n}{\alpha}\left\{\left(\frac{V_{t}^{\alpha, n, B}(0)}{V_{t}^{\alpha, n, 0}(0)}\right)^{\frac{1}{n}}-1\right\} .
$$

Remark that, by Proposition 4.4 of GR and Assumption 2.3, we have

$$
\begin{aligned}
V_{t}^{\alpha, n, 0}(0) & =\operatorname{essup}_{\vartheta \in \Theta_{0}^{(n)}} E\left[U_{\alpha, n}\left(0, G_{t, T}(\vartheta), 0\right) \mid \mathcal{F}_{t}\right] \\
& =\operatorname{essup}_{\vartheta \in \Theta^{n+1}(P)} E\left[-\left(1-\frac{\alpha}{n} G_{t, T}(\vartheta)\right)^{n+1} \mid \mathcal{F}_{t}\right] \\
& =-E\left[\left(1+\frac{{ }^{t} f_{T}^{(n)}}{n}\right)^{n+1} \mid \mathcal{F}_{t}\right] .
\end{aligned}
$$

Recall $Q^{(n)} \in \mathcal{M}_{1+\frac{1}{n}}^{e}(P)$, so that, ${ }^{t} f_{T}^{(n)}>-n$ holds. Hence, $V_{t}^{\alpha, n, 0}(0)<0$. For any $Q \in \mathcal{M}_{1+\frac{1}{n}}^{e}\left(P^{n, B}\right)$, we denote

$$
Z_{t, T}^{Q}:=\frac{Z_{T}^{Q}}{Z_{t}^{Q}}, \quad \text { and } \quad Z_{t}^{Q}:=E\left[\frac{d Q}{d P} \mid \mathcal{F}_{t}\right]
$$

Moreover, we define

$$
\widetilde{V}_{t}^{\alpha, n, B}:=\operatorname{essinf}_{Q \in \mathcal{M}_{1+\frac{1}{n}}^{e}\left(P^{n, B}\right)} E_{Q}\left[\left(Z_{t, T}^{Q}\right)^{\frac{1}{n}}\left(1+\frac{\alpha}{n} B\right)^{-1} \mid \mathcal{F}_{t}\right] .
$$

Remark that we have

$$
\widetilde{V}_{t}^{\alpha, n, 0}=\operatorname{essinf}_{Q \in \mathcal{M}_{1+\frac{1}{n}}^{e}(P)} E_{Q}\left[\left(Z_{t, T}^{Q}\right)^{\frac{1}{n}} \mid \mathcal{F}_{t}\right]=\left(C_{t}^{(n)}\right)^{\frac{1}{n}}
$$

In order to investigate basic properties, we need to show a duality relationship between a portfolio optimization problem and an optimization problem with respect to equivalent martingale measures.

Theorem 4.1 We have the following duality relationship:

$$
\begin{aligned}
& \operatorname{esssup}_{\vartheta \in \Theta_{B}^{(n)}} E\left[-\left(1-\frac{\alpha}{n} G_{t, T}(\vartheta)\right)^{n+1}\left(1+\frac{\alpha}{n} B\right)^{n} \mid \mathcal{F}_{t}\right] \\
& =-\left\{\operatorname{essinf}_{Q \in \mathcal{M}_{1+\frac{1}{n}}^{e}\left(P^{n, B}\right)} E_{Q}\left[\left(Z_{t, T}^{Q}\right)^{\frac{1}{n}}\left(1+\frac{\alpha}{n} B\right)^{-1} \mid \mathcal{F}_{t}\right]\right\}^{-n}(4.1 .)
\end{aligned}
$$

Proof. Firstly, we calculate the left hand side of (4.1) as follows:

$$
\operatorname{LHS} \text { of }(4.1)=-\frac{1}{C_{t}^{n, B}} \operatorname{essinf}_{\vartheta \in \Theta_{B}^{(n)}} E_{P^{n, B}}\left[\left(1-\frac{\alpha}{n} G_{t, T}(\vartheta)\right)^{n+1} \mid \mathcal{F}_{t}\right]
$$




$$
\begin{aligned}
& =-\frac{1}{C_{t}^{n, B}} E_{P^{n, B}}\left[\left(1+\frac{{ }^{t} f_{T}^{(n), B}}{n}\right)^{n+1} \mid \mathcal{F}_{t}\right] \\
& =-\frac{1}{C_{t}^{n, B}} \frac{1}{C_{t}^{(n), B}} E_{Q^{(n), B}}\left[\left(1+\frac{{ }^{t} f_{T}^{(n), B}}{n}\right) \mid \mathcal{F}_{t}\right] \\
& =-\frac{1}{C_{t}^{n, B}} \frac{1}{C_{t}^{(n), B}} .
\end{aligned}
$$

The second equality owes to Proposition 4.4 of GR. On the other hand, we have

RHS of (4.1)

$$
\begin{aligned}
& =-\left\{\operatorname{essinf}_{Q \in \mathcal{M}_{1+\frac{1}{n}}^{e}\left(P^{n, B}\right)} E\left[\left(Z_{t, T}^{Q, n, B} Z_{t, T}^{n, B}\right)^{1+\frac{1}{n}}\left(Z_{t, T}^{n, B}\right)^{-\frac{1}{n}}\left(C_{t}^{n, B}\right)^{\frac{1}{n}} \mid \mathcal{F}_{t}\right]\right\}^{-n} \\
& =-\frac{1}{C_{t}^{n, B}}\left\{\operatorname{essinf}_{Q \in \mathcal{M}_{1+\frac{1}{n}}^{e}\left(P^{n, B}\right)} E_{P^{n, B}}\left[\left(Z_{t, T}^{Q, n, B}\right)^{1+\frac{1}{n}} \mid \mathcal{F}_{t}\right]\right\}^{-n} \\
& =-\frac{1}{C_{t}^{n, B}}\left\{E_{Q^{(n), B}}\left[\left(Z_{t, T}^{(n), B}\right)^{\frac{1}{n}} \mid \mathcal{F}_{t}\right]\right\}^{-n} \\
& =-\frac{1}{C_{t}^{n, B}} \frac{1}{C_{t}^{(n), B}}
\end{aligned}
$$

where

$$
Z_{t, T}^{Q, n, B}:=\frac{Z_{T}^{Q, n, B}}{Z_{t}^{Q, n, B}} \quad \text { and } \quad Z_{t}^{Q, n, B}:=E_{P^{n, B}}\left[\frac{d Q}{d P^{n, B}} \mid \mathcal{F}_{t}\right]
$$

This completes the proof of Theorem 4.1.

Theorem 4.1 provides the following representation of $C_{t}^{\alpha, n}(B)$ :

Corollary 4.2 By the result of Theorem 4.1, we obtain

$$
V_{t}^{\alpha, n, B}(0)=-\left(\widetilde{V}_{t}^{\alpha, n, B}\right)^{-n}
$$

and

$$
C_{t}^{\alpha, n}(B)=\frac{n}{\alpha}\left\{\frac{\widetilde{V}_{t}^{\alpha, n, 0}}{\widetilde{V}_{t}^{\alpha, n, B}}-1\right\} .
$$

Next, we study basic properties of $C_{t}^{\alpha, n}(B)$ by using the above duality relation. First of all, we introduce the basic properties of the EUIV, which have been proved in MS.

Proposition 4.3 (Proposition 4 of MS) We assume that $B$ and $B^{\prime}$ are bounded (not necessarily positive). For fixed $t \in[0, T]$ and $\alpha>0, C_{t}^{\alpha, \exp }(B)$ has the following properties: 
(1) $-\|B\|_{\infty} \leq C_{t}^{\alpha, \exp }(B) \leq\|B\|_{\infty}$,

(2) if $B \leq B^{\prime}$, then $C_{t}^{\alpha, \exp }(B) \leq C_{t}^{\alpha, \exp }\left(B^{\prime}\right)$,

(3) $C_{t}^{\alpha, n}\left(\lambda B+(1-\lambda) B^{\prime}\right) \leq \lambda C_{t}^{\alpha, n}(B)+(1-\lambda) C_{t}^{\alpha, n}\left(B^{\prime}\right)$, for any $\lambda \in[0,1]$,

(4) $C_{t}^{\alpha, n}\left(B+x_{t}\right)=C_{t}^{\alpha, n}(B)+x_{t}$, for any $x_{t} \in \mathcal{L}^{\infty}\left(\mathcal{F}_{t}\right)$.

MS called $C_{t}^{\alpha, \exp }(B)$ a convex monetary utility functional. Furthermore, they remarked that $C_{t}^{\alpha, \exp }(-B)$ is close related to a convex monetary risk measure (see Cheridito, Delbaen and Kupper (2004)).

In order to see that our new valuation $C_{t}^{\alpha, n}(B)$ is available as one of approximate approaches to the EUIV, we wish to prove that $C_{t}^{\alpha, n}(B)$ satisfies Proposition 4.3. Henceforth, we shall prove that this fact holds approximately. Firstly, we obtain the following result being related to (1) and (2) of Proposition 4.3 .

Proposition 4.4 For any $t \in[0, T]$, we have the following:

(1) for $B \in \mathcal{L}_{+}^{\infty}(P), 0 \leq C_{t}^{\alpha, n}(B) \leq\|B\|_{\infty}$,

(2) under Assumptions 2.1 and 2.5 for $B^{\prime}, B \leq B^{\prime} \Longrightarrow C_{t}^{\alpha, n}(B) \leq C_{t}^{\alpha, n}\left(B^{\prime}\right)$.

Proof.

$$
\begin{aligned}
\frac{V_{t}^{\alpha, n, B}(0)}{V_{t}^{\alpha, n, 0}(0)} & =\frac{\operatorname{esssup}_{\vartheta \in \Theta_{B}^{(n)}} E\left[-\left(1-\frac{\alpha}{n} G_{t, T}(\vartheta)\right)^{n+1}\left(1+\frac{\alpha}{n} B\right)^{n} \mid \mathcal{F}_{t}\right]}{\operatorname{esssup}_{\vartheta \in \Theta_{0}^{(n)}} E\left[-\left(1-\frac{\alpha}{n} G_{t, T}(\vartheta)\right)^{n+1} \mid \mathcal{F}_{t}\right]} \\
& \leq\left(1+\frac{\alpha}{n}\|B\|_{\infty}\right)^{n} .
\end{aligned}
$$

By the same way, $B \geq 0$ implies

$$
V_{t}^{\alpha, n, B}(0) / V_{t}^{\alpha, n, 0}(0) \geq 1 .
$$

Hence, we have $0 \leq C_{t}^{\alpha, n}(B) \leq\|B\|_{\infty}$.

(2) Firstly, we obtain $\Theta_{B^{\prime}}^{(n)} \subset \Theta_{B}^{(n)}$, since

$$
\begin{aligned}
\vartheta \in \Theta_{B^{\prime}}^{(n)} & \Rightarrow G_{T}(\vartheta) \in \mathcal{L}^{n+1}\left(P^{n, B^{\prime}}\right) \Rightarrow E\left[G_{T}^{n+1}(\vartheta)\left(1+\frac{\alpha}{n} B^{\prime}\right)^{n}\right]<\infty \\
& \Rightarrow E\left[G_{T}^{n+1}(\vartheta)\left(1+\frac{\alpha}{n} B\right)^{n}\right]<\infty \Rightarrow G_{T}(\vartheta) \in \mathcal{L}^{n+1}\left(P^{n, B}\right) \Rightarrow \vartheta \in \Theta_{B}^{(n)} .
\end{aligned}
$$

Remark that the last inclusion is derived from Theorem 4.12 of of Choulli, Krawczyk and Stricker (1999). Thus, we have

$$
\begin{aligned}
\frac{V_{t}^{\alpha, n, B}(0)}{V_{t}^{\alpha, n, 0}(0)} & =\frac{\operatorname{esssup}_{\vartheta \in \Theta_{B}^{(n)}} E\left[-\left(1-\frac{\alpha}{n} G_{t, T}(\vartheta)\right)^{n+1}\left(1+\frac{\alpha}{n} B\right)^{n} \mid \mathcal{F}_{t}\right]}{\operatorname{esssup}_{\vartheta \in \Theta_{0}^{(n)}} E\left[-\left(1-\frac{\alpha}{n} G_{t, T}(\vartheta)\right)^{n+1} \mid \mathcal{F}_{t}\right]} \\
& \leq \frac{\operatorname{essinf}_{\vartheta \in \Theta_{B}^{(n)}} E\left[\left(1-\frac{\alpha}{n} G_{t, T}(\vartheta)\right)^{n+1}\left(1+\frac{\alpha}{n} B^{\prime}\right)^{n} \mid \mathcal{F}_{t}\right]}{\operatorname{essinf}_{\vartheta \in \Theta_{0}^{(n)}} E\left[\left(1-\frac{\alpha}{n} G_{t, T}(\vartheta)\right)^{n+1} \mid \mathcal{F}_{t}\right]}
\end{aligned}
$$




$$
\begin{aligned}
& \leq \frac{\operatorname{essinf}_{\vartheta \in \Theta_{B^{\prime}}^{(n)}} E\left[\left(1-\frac{\alpha}{n} G_{t, T}(\vartheta)\right)^{n+1}\left(1+\frac{\alpha}{n} B^{\prime}\right)^{n} \mid \mathcal{F}_{t}\right]}{\operatorname{essinf}_{\vartheta \in \Theta_{0}^{(n)}} E\left[\left(1-\frac{\alpha}{n} G_{t, T}(\vartheta)\right)^{n+1} \mid \mathcal{F}_{t}\right]} \\
& =\frac{V_{t}^{\alpha, n, B^{\prime}}(0)}{V_{t}^{\alpha, n, 0}(0)} .
\end{aligned}
$$

This completes the proof of (2) of Proposition 4.4.

Second, we deal with (3) of Proposition 4.3. We define

$$
\bar{V}_{t}^{\alpha, n, B}:=\operatorname{essinf}_{Q \in \mathcal{M}_{1+\frac{1}{n}}^{e}\left(P^{n, B}\right)} E_{Q}\left[\left(Z_{t, T}^{Q}\right)^{\frac{1}{n}}\left(1-\frac{\alpha}{n} B\right) \mid \mathcal{F}_{t}\right] .
$$

Set $\bar{B}:=B /\left(1+\frac{\alpha}{n} B\right) \leq B$. Since we have

$$
1-\frac{\alpha}{n} \bar{B}=\left(1+\frac{\alpha}{n} B\right)^{-1}
$$

we obtain

$$
\widetilde{V}_{t}^{\alpha, n, B}=\bar{V}_{t}^{\alpha, n, \bar{B}} \geq \bar{V}_{t}^{\alpha, n, B}
$$

Now, we denote

$$
\widetilde{C}_{t}^{\alpha, n}(B):=\frac{n}{\alpha}\left\{\frac{\widetilde{V}_{t}^{\alpha, n, 0}}{\bar{V}_{t}^{\alpha, n, B}}-1\right\} .
$$

We prove, on account, that $\widetilde{C}_{t}^{\alpha, n}(B)$ satisfies (3) of Proposition 4.3.

Proposition 4.5 For fixed $t \in[0, T]$, any $\lambda \in[0,1]$ and $B, B^{\prime} \in \mathcal{L}_{+}^{\infty}(P)$ such that $\|B\|_{\infty},\left\|B^{\prime}\right\|_{\infty} \leq n / \alpha$, we have

$$
\widetilde{C}_{t}^{\alpha, n}(B)\left(\lambda B+(1-\lambda) B^{\prime}\right) \leq \lambda \widetilde{C}_{t}^{\alpha, n}(B)+(1-\lambda) \widetilde{C}_{t}^{\alpha, n}\left(B^{\prime}\right) .
$$

Proof. Remark that, for $B \in \mathcal{L}_{+}^{\infty}(P), \mathcal{M}_{1+\frac{1}{n}}^{e}\left(P^{n, B}\right)=\mathcal{M}_{1+\frac{1}{n}}^{e}(P)$ holds. We have only to prove that

$$
\left(\bar{V}^{\alpha, n, \lambda B+(1-\lambda) B^{\prime}}\right)^{-1} \leq \lambda\left(\bar{V}_{t}^{\alpha, n, B}\right)^{-1}+(1-\lambda)\left(\bar{V}^{\alpha, n, B^{\prime}}\right)^{-1}
$$

By the convexity of $1 / x$, we have

$$
\begin{aligned}
& \left(\bar{V}^{\alpha, n, \lambda B+(1-\lambda) B^{\prime}}\right)^{-1} \\
& \quad=\left\{\operatorname{essinf}_{Q \in \mathcal{M}_{1+\frac{1}{n}}^{e}(P)} E_{Q}\left[\left(Z_{t, T}^{Q}\right)^{\frac{1}{n}}\left(\lambda\left(1-\frac{\alpha}{n} B\right)+(1-\lambda)\left(1-\frac{\alpha}{n} B^{\prime}\right)\right) \mid \mathcal{F}_{t}\right]\right\}^{-1} \\
& \leq\left\{\lambda \bar{V}_{t}^{\alpha, n, B}+(1-\lambda) \bar{V}_{t}^{\alpha, n, B^{\prime}}\right\}^{-1} \\
& \leq \frac{\lambda}{\bar{V}_{t}^{\alpha, n, B}}+\frac{1-\lambda}{\bar{V}_{t}^{\alpha, n, B^{\prime}}} .
\end{aligned}
$$


Thus, Proposition 4.5 follows.

Now, in order to see that $C_{t}^{\alpha, n}(B)$ satisfies (3) of Proposition 4.3 approximately, we prove that, for any sufficient large $n, \widetilde{C}_{t}^{\alpha, n}(B)$ is very near to $C_{t}^{\alpha, n}(B)$.

Proposition 4.6 For any sufficient large n, there exists a $C>0$ depending on $\|B\|_{\infty}$ such that

$$
\sup _{0 \leq t \leq T}\left|\widetilde{C}_{t}^{\alpha, n}(B)-C_{t}^{\alpha, n}(B)\right| \leq C \frac{\alpha}{n} .
$$

Proof. Remark that we have

$$
\widetilde{C}_{t}^{\alpha, n}(B)-C_{t}^{\alpha, n}(B)=\frac{n}{\alpha} \frac{\widetilde{V}_{t}^{\alpha, n, 0}}{\bar{V}_{t}^{\alpha, n, B}} \frac{\widetilde{V}_{t}^{\alpha, n, B}-\bar{V}_{t}^{\alpha, n, B}}{\widetilde{V}_{t}^{\alpha, n, B}} .
$$

Firstly, we have the following estimation:

$$
\begin{aligned}
\frac{\widetilde{V}_{t}^{\alpha, n, 0}}{\bar{V}_{t}^{\alpha, n, B}} & =\frac{\widetilde{V}_{t}^{\alpha, n, 0}}{\operatorname{essinf}_{Q \in \mathcal{M}_{1+\frac{1}{n}}^{e}(P)} E_{Q}\left[\left(Z_{t, T}^{Q}\right)^{\frac{1}{n}}\left(1-\frac{\alpha}{n} B\right) \mid \mathcal{F}_{t}\right]} \\
& \leq \frac{\widetilde{V}_{t}^{\alpha, n, 0}}{\left(1-\frac{\alpha}{n}\|B\|_{\infty}\right) \operatorname{essinf}_{Q \in \mathcal{M}_{1+\frac{1}{n}}^{e}(P)} E_{Q}\left[\left(Z_{t, T}^{Q}\right)^{\frac{1}{n}} \mid \mathcal{F}_{t}\right]} \\
& \leq\left(1-\frac{\alpha}{n}\|B\|_{\infty}\right)^{-1} .
\end{aligned}
$$

Remark that, for any sufficient large $n, 1-\frac{\alpha}{n}\|B\|_{\infty}>0$ holds. Moreover, we have

$$
\begin{aligned}
\widetilde{V}_{t}^{\alpha, n, B}-\bar{V}_{t}^{\alpha, n, B} & \operatorname{essinf}_{Q \in \mathcal{M}_{1+\frac{1}{n}}^{e}(P)} E_{Q}\left[\left(Z_{t, T}^{Q}\right)^{\frac{1}{n}}\left(1+\frac{\alpha}{n} B\right)^{-1} \mid \mathcal{F}_{t}\right] \\
& -\operatorname{essinf}_{Q \in \mathcal{M}_{1+\frac{1}{n}}^{e}(P)} E_{Q}\left[\left(Z_{t, T}^{Q}\right)^{\frac{1}{n}}\left(1-\frac{\alpha}{n} B\right) \mid \mathcal{F}_{t}\right] \\
= & \operatorname{essinf}_{Q \in \mathcal{M}_{1+\frac{1}{n}}^{e}(P)} E_{Q}\left[\left(Z_{t, T}^{Q}\right)^{\frac{1}{n}}\left(1-\frac{\alpha}{n} B+\left(\frac{\alpha}{n} B\right)^{2}\left(1+\frac{\alpha}{n} B\right)^{-1}\right) \mid \mathcal{F}_{t}\right] \\
& -\operatorname{essinf}_{Q \in \mathcal{M}_{1+\frac{1}{n}}^{e}(P)} E_{Q}\left[\left(Z_{t, T}^{Q}\right)^{\frac{1}{n}}\left(1-\frac{\alpha}{n} B\right) \mid \mathcal{F}_{t}\right] \\
\leq & E_{\tilde{Q}}\left[\left(Z_{t, T}^{\tilde{Q}}\right)^{\frac{1}{n}}\left(1-\frac{\alpha}{n} B+\left(\frac{\alpha}{n} B\right)^{2}\left(1+\frac{\alpha}{n} B\right)^{-1}\right) \mid \mathcal{F}_{t}\right] \\
& -E_{\tilde{Q}}\left[\left(Z_{t, T}^{\widetilde{Q}}\right)^{\frac{1}{n}}\left(1-\frac{\alpha}{n} B\right) \mid \mathcal{F}_{t}\right] \\
\leq & \left(\frac{\alpha}{n}\|B\|_{\infty}\right)^{2} E_{\tilde{Q}}\left[\left(Z_{t, T}^{\tilde{Q}}\right)^{\frac{1}{n}}\left(1+\frac{\alpha}{n} B\right)^{-1} \mid \mathcal{F}_{t}\right]
\end{aligned}
$$


where $\widetilde{Q}$ is given by

$\operatorname{essinf}_{Q \in \mathcal{M}_{1+\frac{1}{n}}^{e}(P)} E_{Q}\left[\left(Z_{t, T}^{Q}\right)^{\frac{1}{n}}\left(1-\frac{\alpha}{n} B\right) \mid \mathcal{F}_{t}\right]=E_{\tilde{Q}}\left[\left(Z_{t, T}^{\widetilde{Q}}\right)^{\frac{1}{n}}\left(1-\frac{\alpha}{n} B\right) \mid \mathcal{F}_{t}\right]$.

On the other hand, we have

$$
\begin{aligned}
\widetilde{V}_{t}^{\alpha, n, B} & =\operatorname{essinf}_{Q \in \mathcal{M}_{1+\frac{1}{n}}^{e}(P)} E_{Q}\left[\left(Z_{t, T}^{Q}\right)^{\frac{1}{n}}\left(1+\frac{\alpha}{n} B\right)^{-1} \mid \mathcal{F}_{t}\right] \\
& \geq \operatorname{essinf}_{Q \in \mathcal{M}_{1+\frac{1}{n}}^{e}(P)} E_{Q}\left[\left(Z_{t, T}^{Q}\right)^{\frac{1}{n}}\left(1-\frac{\alpha}{n} B\right) \mid \mathcal{F}_{t}\right] \\
& =E_{\tilde{Q}}\left[\left(Z_{t, T}^{\widetilde{Q}}\right)^{\frac{1}{n}}\left(1-\frac{\alpha}{n} B\right) \mid \mathcal{F}_{t}\right] .
\end{aligned}
$$

By (4.4) and (4.5),

$$
\begin{aligned}
\frac{\widetilde{V}_{t}^{\alpha, n, B}-\bar{V}_{t}^{\alpha, n, B}}{\widetilde{V}_{t}^{\alpha, n, B}} & \leq \frac{\left(\frac{\alpha}{n}\|B\|_{\infty}\right)^{2} E_{\tilde{Q}}\left[\left(Z_{t, T}^{\widetilde{Q}}\right)^{\frac{1}{n}}\left(1+\frac{\alpha}{n} B\right)^{-1} \mid \mathcal{F}_{t}\right]}{E_{\widetilde{Q}}\left[\left(Z_{t, T}^{\tilde{Q}}\right)^{\frac{1}{n}}\left(1-\frac{\alpha}{n} B\right) \mid \mathcal{F}_{t}\right]} \\
& \leq \frac{\left(\frac{\alpha}{n}\|B\|_{\infty}\right)^{2}}{1-\frac{\alpha}{n}\|B\|_{\infty}} .
\end{aligned}
$$

As a result, by $(4.2),(4.3)$ and (4.6), we obtain, together with $\widetilde{C}_{t}^{\alpha, n}(B) \geq$ $C_{t}^{\alpha, n}(B)$

$$
\sup _{0 \leq t \leq T}\left|\widetilde{C}_{t}^{\alpha, n}(B)-C_{t}^{\alpha, n}(B)\right| \leq \frac{n}{\alpha} \frac{\left(\frac{\alpha}{n}\|B\|_{\infty}\right)^{2}}{\left(1-\frac{\alpha}{n}\|B\|_{\infty}\right)^{2}} \leq C \frac{\alpha}{n} .
$$

By using Proposition 4.6 together with Proposition 4.5, we obtain that $C_{t}^{\alpha, n}(B)$ is $\mathcal{F}_{t}$-measurably convex in $B$ approximately.

Corollary 4.7 Suppose that $\lambda \in[0,1]$ and $B, B^{\prime} \in \mathcal{L}_{+}^{\infty}(P)$. For fixed $t \in[0, T]$ and any sufficient large $n$, there exists a constant $C>0$ depending on $\|B\|_{\infty}$ and $\left\|B^{\prime}\right\|_{\infty}$ such that

$$
C_{t}^{\alpha, n}\left(\lambda B+(1-\lambda) B^{\prime}\right) \leq \lambda C_{t}^{\alpha, n}(B)+(1-\lambda) C_{t}^{\alpha, n}\left(B^{\prime}\right)+C \frac{\alpha}{n} .
$$

Proof. Remark that, for $B \in \mathcal{L}_{+}^{\infty}(P), \widetilde{V}_{t}^{\alpha, n, B} \geq \bar{V}_{t}^{\alpha, n, B}$. By Propositions 4.5 and 4.6, we have

$$
\begin{aligned}
C_{t}^{\alpha, n}\left(\lambda B+(1-\lambda) B^{\prime}\right) & \leq \widetilde{C}_{t}^{\alpha, n}\left(\lambda B+(1-\lambda) B^{\prime}\right) \\
& \leq \lambda \widetilde{C}_{t}^{\alpha, n}(B)+(1-\lambda) \widetilde{C}_{t}^{\alpha, n}\left(B^{\prime}\right) \\
& \leq \lambda\left(C_{t}^{\alpha, n}(B)+C \frac{\alpha}{n}\right)+(1-\lambda)\left(C_{t}^{\alpha, n}\left(B^{\prime}\right)+C \frac{\alpha}{n}\right) \\
& =\lambda C_{t}^{\alpha, n}(B)+(1-\lambda) C_{t}^{\alpha, n}\left(B^{\prime}\right)+C \frac{\alpha}{n} .
\end{aligned}
$$


Thirdly, we concentrate on (4) of Proposition 4.3. By a similar way with the above, we prove the following:

Proposition 4.8 Let $x_{t}$ be a bounded $\mathcal{F}_{t}$-measurable random variable. For fixed $t \in[0, T]$ and any sufficient large $n$, there exists a constant $C>0$ depending on $\left\|x_{t}\right\|_{\infty}$ and $\|B\|_{\infty}$ such that

$$
\left|C_{t}^{\alpha, n}\left(B+x_{t}\right)-C_{t}^{\alpha, n}(B)-x_{t}\right| \leq C \frac{\alpha}{n} .
$$

Proof. We prove firstly the case where $C_{t}^{\alpha, n}\left(B+x_{t}\right)-C_{t}^{\alpha, n}(B)-x_{t} \geq 0$. Remark that

$$
C_{t}^{\alpha, n}\left(B+x_{t}\right)-C_{t}^{\alpha, n}(B) \leq \frac{n}{\alpha} \widetilde{V}_{t}^{\alpha, n, 0} \frac{\widetilde{V}_{t}^{\alpha, n, B}-\bar{V}_{t}^{\alpha, n, B+x_{t}}}{\widetilde{V}_{t}^{\alpha, n, B} \bar{V}_{t}^{\alpha, n, B+x_{t}}} .
$$

Firstly, we have

$$
\begin{aligned}
\widetilde{V}_{t}^{\alpha, n, B}-\bar{V}_{t}^{\alpha, n, B+x_{t}} & \\
= & \operatorname{essinf}_{Q \in \mathcal{M}_{1+\frac{1}{n}}^{e}(P)} E_{Q}\left[\left(Z_{t, T}^{Q}\right)^{\frac{1}{n}}\left(1+\frac{\alpha}{n} B\right)^{-1} \mid \mathcal{F}_{t}\right] \\
& -\operatorname{essinf}_{Q \in \mathcal{M}_{1+\frac{1}{n}}^{e}(P)} E_{Q}\left[\left(Z_{t, T}^{Q}\right)^{\frac{1}{n}}\left(1-\frac{\alpha}{n}\left(B+x_{t}\right)\right) \mid \mathcal{F}_{t}\right] \\
\leq & E_{\tilde{Q}}\left[\left(Z_{t, T}^{\tilde{Q}}\right)^{\frac{1}{n}}\left(1+\frac{\alpha}{n} B\right)^{-1} \mid \mathcal{F}_{t}\right]-E_{\tilde{Q}}\left[\left(Z_{t, T}^{\tilde{Q}}\right)^{\frac{1}{n}}\left(1-\frac{\alpha}{n}\left(B+x_{t}\right)\right) \mid \mathcal{F}_{t}\right] \\
\leq & \left(\left(\frac{\alpha}{n}\|B\|_{\infty}\right)^{2}+\frac{\alpha}{n} x_{t}\right) E_{\tilde{Q}}\left[\left(Z_{t, T}^{\tilde{Q}}\right)^{\frac{1}{n}} \mid \mathcal{F}_{t}\right],
\end{aligned}
$$

where $\widetilde{Q} \in \mathcal{M}_{1+\frac{1}{n}}^{e}(P)$ is given by

$$
\begin{aligned}
& \operatorname{essinf}_{Q \in \mathcal{M}_{1+\frac{1}{n}}^{e}(P)} E_{Q}\left[\left(Z_{t, T}^{Q}\right)^{\frac{1}{n}}\left(1-\frac{\alpha}{n}\left(B+x_{t}\right)\right) \mid \mathcal{F}_{t}\right] \\
& =E_{\widetilde{Q}}\left[\left(Z_{t, T}^{\bar{Q}}\right)^{\frac{1}{n}}\left(1-\frac{\alpha}{n}\left(B+x_{t}\right)\right) \mid \mathcal{F}_{t}\right] .
\end{aligned}
$$

The last inequality of (4.9) is given from

$$
\left(1+\frac{\alpha}{n} B\right)^{-1}-1+\frac{\alpha}{n} B=\left(\frac{\alpha}{n} B\right)^{2}\left(1+\frac{\alpha}{n} B\right)^{-1} \leq\left(\frac{\alpha}{n} B\right)^{2} .
$$

Next, we have

$$
\begin{aligned}
& \widetilde{V}_{t}^{\alpha, n, B} \bar{V}_{t}^{\alpha, n, B+x_{t}} \\
& \quad=\operatorname{essinf}_{Q \in \mathcal{M}_{1+\frac{1}{n}}^{e}(P)} E_{Q}\left[\left(Z_{t, T}^{Q}\right)^{\frac{1}{n}}\left(1+\frac{\alpha}{n} B\right)^{-1} \mid \mathcal{F}_{t}\right] E_{\tilde{Q}}\left[\left(Z_{t, T}^{\widetilde{Q}}\right)^{\frac{1}{n}}\left(1-\frac{\alpha}{n}\left(B+x_{t}\right)\right) \mid \mathcal{F}_{t}\right] \\
& \quad \geq\left(1+\frac{\alpha}{n}\|B\|_{\infty}\right)^{-1} \widetilde{V}_{t}^{\alpha, n, 0}\left(1-\frac{\alpha}{n}\left(\|B\|_{\infty}+\left\|x_{t}\right\|_{\infty}\right)\right) E_{\tilde{Q}}\left[\left(Z_{t, T}^{\widetilde{Q}}\right)^{\frac{1}{n}} \mid \mathcal{F}_{t}\right] .
\end{aligned}
$$


Thus, we can conclude

RHS of (4.8)

$$
\begin{aligned}
& \leq \frac{n}{\alpha} \widetilde{V}_{t}^{\alpha, n, 0} \frac{\left(\left(\frac{\alpha}{n}\|B\|_{\infty}\right)^{2}+\frac{\alpha}{n} x_{t}\right) E_{\tilde{Q}}\left[\left(Z_{t, T}^{\tilde{Q}}\right)^{\frac{1}{n}} \mid \mathcal{F}_{t}\right]}{\left(1+\frac{\alpha}{n}\|B\|_{\infty}\right)^{-1}\left(1-\frac{\alpha}{n}\left(\|B\|_{\infty}+\left\|x_{t}\right\|_{\infty}\right)\right) E_{\tilde{Q}}\left[\left(Z_{t, T}^{\tilde{Q}}\right)^{\frac{1}{n}} \mid \mathcal{F}_{t}\right] \widetilde{V}_{t}^{\alpha, n, 0}} \\
& =\frac{\left(\frac{\alpha}{n}\|B\|_{\infty}^{2}+x_{t}\right)\left(1+\frac{\alpha}{n}\|B\|_{\infty}\right)}{1-\frac{\alpha}{n}\left(\|B\|_{\infty}+\left\|x_{t}\right\|_{\infty}\right)}
\end{aligned}
$$

On the other hand,

$$
\frac{1+\frac{\alpha}{n}\|B\|_{\infty}}{1-\frac{\alpha}{n}\left(\|B\|_{\infty}+\left\|x_{t}\right\|_{\infty}\right)}=1+\frac{\alpha}{n} \frac{2\|B\|_{\infty}+\left\|x_{t}\right\|_{\infty}}{1-\frac{\alpha}{n}\left(\|B\|_{\infty}+\left\|x_{t}\right\|_{\infty}\right)} \leq 1+C \frac{\alpha}{n} .
$$

(4.10) and (4.11) imply (4.7) for the case where $C_{t}^{\alpha, n}\left(B+x_{t}\right)-C_{t}^{\alpha, n}(B)-x_{t} \geq 0$.

Next, we treat the reverse case. Without loss of generality, we assume that $x_{t}$ is positive. Remark that

$$
\left(1+\frac{\alpha}{n}\left(B+x_{t}\right)\right)^{-1} \leq 1-\frac{\alpha}{n}\left(B+x_{t}\right)+\frac{\alpha^{2}}{n^{2}}\left(B+x_{t}\right)^{2} .
$$

Since we can prove

$$
\bar{V}_{t}^{\alpha, n, B}-\widetilde{V}_{t}^{\alpha, n, B+x_{t}} \geq\left(\frac{\alpha}{n} x_{t}-2 \frac{\alpha^{2}}{n^{2}}\left(\|B\|_{\infty}^{2}+\left\|x_{t}\right\|_{\infty}^{2}\right)\right) E_{\tilde{Q}}\left[\left(Z_{t, T}^{\tilde{Q}}\right)^{\frac{1}{n}} \mid \mathcal{F}_{t}\right],
$$

where $\widetilde{Q} \in \mathcal{M}_{1+\frac{1}{n}}^{e}(P)$ is given by

$$
\begin{aligned}
& \operatorname{essinf}_{Q \in \mathcal{M}_{1+\frac{1}{n}}^{e}(P)} E_{Q}\left[\left(Z_{t, T}^{Q}\right)^{\frac{1}{n}}\left(1-\frac{\alpha}{n} B\right) \mid \mathcal{F}_{t}\right] \\
& =E_{\tilde{Q}}\left[\left(Z_{t, T}^{\tilde{Q}}\right)^{\frac{1}{n}}\left(1-\frac{\alpha}{n} B\right) \mid \mathcal{F}_{t}\right]
\end{aligned}
$$

and

$$
\bar{V}_{t}^{\alpha, n, B} \widetilde{V}_{t}^{\alpha, n, B+x_{t}} \leq E_{\tilde{Q}}\left[\left(Z_{t, T}^{\tilde{Q}}\right)^{\frac{1}{n}} \mid \mathcal{F}_{t}\right] \widetilde{V}_{t}^{\alpha, n, 0},
$$

there exists a constant $C>0$ such that

$$
\begin{aligned}
C_{t}^{\alpha, n}\left(B+x_{t}\right)-C_{t}^{\alpha, n}(B) & \geq \frac{n}{\alpha} \widetilde{V}_{t}^{\alpha, n, 0} \frac{\bar{V}_{t}^{\alpha, n, B}-\widetilde{V}_{t}^{\alpha, n, B+x_{t}}}{\bar{V}_{t}^{\alpha, n, B} \widetilde{V}_{t}^{\alpha, n, B+x_{t}}} \\
& \geq x_{t}-2 \frac{\alpha}{n}\left(\|B\|_{\infty}^{2}+\left\|x_{t}\right\|_{\infty}^{2}\right) \\
& \geq x_{t}-C \frac{\alpha}{n} .
\end{aligned}
$$

Hence, Proposition 4.8 follows. 


\section{Asymptotic behavior}

We have to study the asymptotic behavior of our new valuation $C_{t}^{\alpha, n}(B)$ as $n$ tends to $\infty$ so as to make sure that $C_{t}^{\alpha, n}(B)$ is justified as an approximate approach to the EUIV. In this section, we prove that $C_{t}^{\alpha, n}(B)$ converges to the EUIV in probability. Remark that GR have proved that the $p$-optimal martingale measure converges to the minimal entropy martingale measure as $p$ tends to 1 . In the proof of the following theorem, this asymptotic behavior will play a vital role.

Theorem 5.1 Suppose that $B \in \mathcal{L}_{+}^{\infty}(P)$. For fixed $t \in[0, T], C_{t}^{\alpha, n}(B)$ converges to $C_{t}^{\alpha, \exp }(B)$ in probability as $n \rightarrow \infty$.

Proof. Step 1 We shall prove that $\frac{V_{t}^{\alpha, n, B}}{V_{t}^{\alpha, n, 0}}$ converges to

$$
\frac{\operatorname{esssup}_{\vartheta \in \Theta_{\exp }} E\left[U_{\alpha, \exp }\left(0, G_{t, T}(\vartheta), B\right) \mid \mathcal{F}_{t}\right]}{\operatorname{esssup}_{\vartheta \in \Theta^{\exp }} E\left[U_{\alpha, \exp }\left(0, G_{t, T}(\vartheta), 0\right) \mid \mathcal{F}_{t}\right]} \text { in probability. }
$$

For small $\varepsilon>0$, there exists a sufficient large odd number $n_{0}$ such that, for any odd number $n \geq n_{0}$,

$$
(1-\varepsilon) e^{\alpha B} \leq e^{\alpha B}-\varepsilon \leq\left(1+\frac{\alpha}{n} B\right)^{n} \leq e^{\alpha B} \quad P \text {-a.s. }
$$

Since $n+1$ is even, we have

$$
\begin{aligned}
& \operatorname{esssup}_{\vartheta \in \Theta_{B}^{(n)}} E\left[-\left(1-\frac{\alpha}{n} G_{t, T}(\vartheta)\right)^{n+1} e^{\alpha B} \mid \mathcal{F}_{t}\right] \leq V_{t}^{\alpha, n, B}(0) \\
& \leq(1-\varepsilon) \operatorname{esssup}_{\vartheta \in \Theta_{B}^{(n)}} E\left[-\left(1-\frac{\alpha}{n} G_{t, T}(\vartheta)\right)^{n+1} e^{\alpha B} \mid \mathcal{F}_{t}\right] .
\end{aligned}
$$

Now, we define a probability measure $P^{\exp , B}$ equivalent to $P$ as

$$
\frac{d P^{\exp , B}}{d P}=C^{\exp , B} e^{\alpha B},
$$

where $C^{\exp , B} \in \mathbf{R}_{+}$. We denote

$$
Z_{t}^{\exp , B}:=E\left[\frac{d P^{\exp , B}}{d P} \mid \mathcal{F}_{t}\right]
$$

and

$$
Z_{t, T}^{\exp , B}:=Z_{T}^{\exp , B} / Z_{t}^{\exp , B}=C_{t}^{\exp , B} e^{\alpha B},
$$

where $C_{t}^{\exp , B}$ is an $\mathcal{F}_{t}$-measurable positive random variable. Then, we have

$\operatorname{LHS}$ of $(5.1)=\frac{1}{C_{t}^{\exp , B}} \operatorname{esssup}_{\vartheta \in \Theta_{B}^{(n)}} E_{P^{\exp , B}}\left[-\left(1-\frac{\alpha}{n} G_{t, T}(\vartheta)\right)^{n+1} \mid \mathcal{F}_{t}\right]$ 


$$
\begin{aligned}
& =-\frac{1}{C_{t}^{\exp , B}} \operatorname{essinf}_{\vartheta \in \Theta^{n+1}\left(P_{\exp , B}\right)} E_{P_{\exp , B}}\left[\left(1-\frac{\alpha}{n} G_{t, T}(\vartheta)\right)^{n+1} \mid \mathcal{F}_{t}\right] \\
& =-\frac{1}{C_{t}^{\exp , B}} E_{P^{\exp , B}}\left[\left(1+\frac{{ }^{t} f_{T}^{(n) \exp , B}}{n}\right)^{n+1} \mid \mathcal{F}_{t}\right]
\end{aligned}
$$

where ${ }^{t} f_{T}^{(n), \exp , B}$ is the projection of "1" onto ${ }^{t} \mathcal{K}_{n+1}\left(P^{\exp , B}\right)$ in $\mathcal{L}^{n+1}\left(P^{\exp , B}\right)$. Remark that, $B \in \mathcal{L}_{+}^{\infty}(P)$ yields

$$
\Theta_{B}^{(n)}=\Theta^{n+1}\left(P^{n, B}\right)=\Theta^{n+1}\left(P^{\exp , B}\right)=\Theta^{n+1}(P) .
$$

By Lemma 4.13 of GR, ${ }^{t} f_{T}^{(n), \exp , B}$ converges in probability to ${ }^{t} f_{T}^{\exp , B}$, which is in $\cap_{n \geq 0}{ }^{t} \mathcal{K}_{n+1}\left(P^{\exp , B}\right)$. Thus, for every sequence $n_{k}$, we can extract a subsequence (still denoted by $n_{k}$ ) such that

$$
{ }^{t} f_{T}^{\left(n_{k}\right), \exp , B} \rightarrow{ }^{t} f_{T}^{\exp , B} \quad P \text {-a.s. . }
$$

Remark that there exists the minimal entropy martingale measure $\bar{P}^{\text {exp }, B}$ for $P^{\exp , B}$, the density process of which satisfies $\mathcal{R}_{L \log L}\left(P^{\exp , B}\right)$. We denote

$$
\bar{Z}_{t}^{\exp , B}:=E_{P_{\exp , B}}\left[\frac{d \bar{P}^{\exp , B}}{d P^{\exp , B}} \mid \mathcal{F}_{t}\right],
$$

and, by the proof of Proposition 4.15 of GR, we can represent

$$
\bar{Z}_{t, T}^{\exp , B}:=\bar{Z}_{T}^{\exp , B} / \bar{Z}_{t}^{\exp , B}=\bar{C}_{t}^{\exp , B} \exp \left({ }^{t} f_{T}^{\exp , B}\right),
$$

where $\bar{C}_{t}^{\exp , B}$ is an $\mathcal{F}_{t}$-measurable positive random variable. In addition, the proof of Proposition 4.15 of GR implies that

$$
\begin{aligned}
& \lim _{n_{k} \rightarrow \infty} E_{P_{\exp , B}}\left[\left(1+\frac{{ }^{t} f_{T}^{\left(n_{k}\right), \exp , B}}{n_{k}}\right)^{n_{k}+1} \mid \mathcal{F}_{t}\right] \\
& =\lim _{n_{k} \rightarrow \infty} E_{P_{\exp , B}}\left[\left(1+\frac{{ }^{t} f_{T}^{\left(n_{k}\right), \exp , B}}{n_{k}}\right)^{n_{k}} \mid \mathcal{F}_{t}\right] \\
& =E_{P_{\exp , B}}\left[\exp \left({ }^{t} f_{T}^{\exp , B}\right) \mid \mathcal{F}_{t}\right] \quad P \text {-a.s. . }
\end{aligned}
$$

Hence, we obtain

$$
E_{P_{\exp , B}}\left[\left(1+\frac{{ }^{t} f_{T}^{(n), \exp , B}}{n}\right)^{n+1} \mid \mathcal{F}_{t}\right] \rightarrow E_{P_{\exp , B}}\left[\exp \left({ }^{t} f_{T}^{\exp , B}\right) \mid \mathcal{F}_{t}\right]
$$

in probability. Moreover, as for the sequence ${ }^{t} f_{T}^{(n)}$, Lemma 4.13 of GR yields that ${ }^{t} f_{T}^{(n)} \rightarrow{ }^{t} f_{T}^{\exp }$ in probability. Besides, ${ }^{t} f_{T}^{\text {exp }}$ is included in $\cap_{n \geq 0}{ }^{t} \mathcal{K}_{n+1}(P)$. 
Remark that the minimal entropy martingale measure $\bar{P}$ for $P$ is given by

$$
\frac{d \bar{P}}{d P}=\bar{C} \exp \left({ }^{0} f_{T}^{\exp }\right)
$$

where $\bar{C} \in \mathbf{R}_{+}$. Hence, the same sort of argument as the above shows

$$
V_{t}^{\alpha, n, 0} \rightarrow-E\left[\exp \left({ }^{t} f_{T}^{\exp }\right) \mid \mathcal{F}_{t}\right] \quad \text { in probability. }
$$

Since $\varepsilon$ is arbitrary, (5.1), (5.2) and (5.3) yield

$$
\frac{V_{t}^{\alpha, n, B}}{V_{t}^{\alpha, n, 0}} \rightarrow \frac{E\left[\exp \left({ }^{t} f_{T}^{\exp , B}\right) e^{\alpha B} \mid \mathcal{F}_{t}\right]}{E\left[\exp \left({ }^{t} f_{T}^{\exp }\right) \mid \mathcal{F}_{t}\right]} \quad \text { in probability . }
$$

Now, we shall prove that

$$
E_{P \exp , B}\left[\exp \left({ }^{t} f_{T}^{\exp , B}\right) \mid \mathcal{F}_{t}\right]=\operatorname{essinf}_{\vartheta \in \Theta^{\exp }} E_{P \exp , B}\left[\exp \left(-\alpha G_{t, T}(\vartheta)\right) \mid \mathcal{F}_{t}\right] .
$$

By Proposition 1 of MS, there exists an $\eta \in \Theta^{\exp }$ such that $G_{t, T}(\eta)={ }^{t} f_{T}^{\exp , B}$. For any $\vartheta \in \Theta^{\exp }$, we denote $-\alpha \vartheta=\bar{\vartheta}+\eta$. Then, we have

$$
\begin{aligned}
& \operatorname{essinf}_{\vartheta \in \Theta^{\exp }} E_{P_{\exp , B}}\left[\exp \left(-\alpha G_{t, T}(\vartheta)\right) \mid \mathcal{F}_{t}\right] \\
& =\operatorname{essinf}_{\bar{\vartheta} \in \Theta^{\exp }} E_{P^{\exp , B}}\left[e^{G_{t, T}(\bar{\vartheta})} e^{G_{t, T}(\eta)} \mid \mathcal{F}_{t}\right] \\
& =\frac{1}{\bar{C}_{t}^{\exp , B}} \operatorname{essinf}_{\bar{\vartheta} \in \Theta^{\exp }} E_{\bar{P}^{\exp , B}}\left[e^{G_{t, T}(\bar{\vartheta})} \mid \mathcal{F}_{t}\right] .
\end{aligned}
$$

Jensen's inequality yields

$$
E_{\bar{P}^{\exp , B}}\left[e^{G_{t, T}(\bar{\vartheta})} \mid \mathcal{F}_{t}\right] \geq \exp \left\{E_{\bar{P}^{\exp , B}}\left[G_{t, T}(\bar{\vartheta}) \mid \mathcal{F}_{t}\right]\right\}=1 .
$$

On the other hand, if we set $\bar{\vartheta} \equiv 0$, then

$$
E_{\bar{P}^{\exp , B}}\left[e^{G_{t, T}(\bar{\vartheta})} \mid \mathcal{F}_{t}\right]=1 .
$$

Hence, we obtain (5.5).

Let us go back to (5.4). We can conclude that, together with (5.5),

$$
\frac{V_{t}^{\alpha, n, B}}{V_{t}^{\alpha, n, 0}} \rightarrow \frac{\operatorname{esssup}_{\vartheta \in \Theta_{\exp }} E\left[U_{\alpha, \exp }\left(0, G_{t, T}(\vartheta), B\right) \mid \mathcal{F}_{t}\right]}{\operatorname{esssup}_{\vartheta \in \Theta^{\exp }} E\left[U_{\alpha, \exp }\left(0, G_{t, T}(\vartheta), 0\right) \mid \mathcal{F}_{t}\right]} \quad \text { in probability . }
$$

Step 2 We prove the following:

Lemma 5.2 Let $A$ be a compact set on $\mathbf{R}$ and $\left\{X_{n}\right\}_{n \geq 1}$ a sequence of $A$-valued random variables such that $X_{n}$ converges to a random variable $X$ in probability. Moreover, suppose that a sequence $f_{n}$ of continuous functions converges to a continuous function $f$ on $A$. Then, we have

$$
f_{n}\left(X_{n}\right) \rightarrow f(X) \quad \text { in probability. }
$$


Proof of Lemma 5.2. We fix an $\varepsilon>0$ arbitrarily. We have only to show

$$
\lim _{n \rightarrow \infty} P\left(\left\{\left|f_{n}\left(X_{n}\right)-f(X)\right|<\varepsilon\right\}\right)=1 .
$$

Now, we calculate the lower bound of the left hand side.

$$
\begin{aligned}
& P\left(\left\{\left|f_{n}\left(X_{n}\right)-f(X)\right|<\varepsilon\right\}\right) \\
&= P\left(\left\{\left|f_{n}\left(X_{n}\right)-f\left(X_{n}\right)+f\left(X_{n}\right)-f(X)\right|<\varepsilon\right\}\right) \\
& \geq P\left(\left\{\left|f_{n}\left(X_{n}\right)-f\left(X_{n}\right)\right|+\left|f\left(X_{n}\right)-f(X)\right|<\varepsilon\right\}\right) \\
& \geq P\left(\left\{\left|f_{n}\left(X_{n}\right)-f\left(X_{n}\right)\right|<\varepsilon / 2\right\} \cap\left\{\left|f\left(X_{n}\right)-f(X)\right|<\varepsilon / 2\right\}\right) \\
&= P\left(\left\{\left|f_{n}\left(X_{n}\right)-f\left(X_{n}\right)\right|<\varepsilon / 2\right\}\right)+P\left(\left\{\left|f\left(X_{n}\right)-f(X)\right|<\varepsilon / 2\right\}\right) \\
&-P\left(\left\{\left|f_{n}\left(X_{n}\right)-f\left(X_{n}\right)\right|<\varepsilon / 2\right\} \cup\left\{\left|f\left(X_{n}\right)-f(X)\right|<\varepsilon / 2\right\}\right) \\
& \geq P\left(\left\{\left|f_{n}\left(X_{n}\right)-f\left(X_{n}\right)\right|<\varepsilon / 2\right\}\right)+P\left(\left\{\left|f\left(X_{n}\right)-f(X)\right|<\varepsilon / 2\right\}\right)-1
\end{aligned}
$$

Since $X_{n} \in A$ for any $n \geq 1 P$-a.s. and $f_{n} \rightarrow f$ on $A$, we have, for any sufficient large $n$,

$$
P\left(\left\{\left|f_{n}\left(X_{n}\right)-f\left(X_{n}\right)\right|<\varepsilon / 2\right\}\right)=1 .
$$

Moreover, there exists a $\delta>0$ such that $|x-y|<\delta \Rightarrow|f(x)-f(y)|<\varepsilon / 2$. Thus,

$$
P\left(\left\{\left|f\left(X_{n}\right)-f(X)\right|<\varepsilon / 2\right\}\right) \geq P\left(\left\{\left|X_{n}-X\right|<\delta\right\}\right) \rightarrow 1 .
$$

This completes the proof of Lemma 5.2

Step 3 We denote

$$
U_{n}:=\frac{V_{t}^{\alpha, n, B}(0)}{V_{t}^{\alpha, n, 0}(0)}
$$

Then, we can represent

$$
C_{t}^{\alpha, n}(B)=\frac{n}{\alpha}\left\{U_{n}^{\frac{1}{n}}-1\right\} .
$$

Furthermore, $U_{n}$ satisfies

$$
1 \leq U_{n} \leq\left(1+\frac{\alpha}{n}\|B\|_{\infty}\right)^{n} \leq e^{\alpha\|B\|_{\infty}} .
$$

By Step 1, $U_{n}$ converges to

$$
\frac{\operatorname{esssup}_{\vartheta \in \Theta^{\exp }} E\left[U_{\alpha, \exp }\left(0, G_{t, T}(\vartheta), B\right) \mid \mathcal{F}_{t}\right]}{\operatorname{esssup}_{\vartheta \in \Theta^{\exp }} E\left[U_{\alpha, \exp }\left(0, G_{t, T}(\vartheta), 0\right) \mid \mathcal{F}_{t}\right]}(=: U)
$$

in probability. Incidentally, MS have proved

$$
C_{t}^{\alpha, \exp }(B)=\frac{1}{\alpha} \log U .
$$


Moreover, $f_{n}(x):=\frac{n}{\alpha}\left\{x^{1 / n}-1\right\}$ converges to $f(x):=\frac{1}{\alpha} \log x$ on the compact set $\left[1, \exp \left\{\alpha\|B\|_{\infty}\right\}\right]$. By Lemma 5.2, we can conclude that

$$
C_{t}^{\alpha, n}(B) \rightarrow C_{t}^{\alpha, \exp }(B) \quad \text { in probability. }
$$

This completes the proof of Theorem 5.1.

\section{Extension to bid valuation}

The definition of the utility indifference valuation is given from view of a seller. In other words, it is a proposition of an asking-price for a contingent claim. Thus, when we try to suggest a bid-price, it is natural that we improve the utility indifference valuation to an adapted process $\bar{C}_{t}(B)$ satisfying

$\operatorname{esssup}_{\vartheta \in \Theta} E\left[U\left(x_{t}+G_{t, T}(\vartheta)\right) \mid \mathcal{F}_{t}\right]=\operatorname{esssup}_{\vartheta \in \Theta} E\left[U\left(x_{t}-\bar{C}_{t}(B)+G_{t, T}(\vartheta)+B\right) \mid \mathcal{F}_{t}\right]$.

In particular, as for the EUIV, its bid valuation is given by $-C_{t}^{\alpha, \exp }(-B)$. Remark that the EUIV is defined for bounded contingent claims which may value in negative numbers. On the other hand, our valuation $C_{t}^{\alpha, n}(B)$ is available for only positive contingent claims. At least, we have to restrict that $B$ has a lower bound in order that $C_{t}^{\alpha, n}(B)$ is well-defined. Hence, we should define a bid valuation other than the ask valuation $C_{t}^{\alpha, n}(B)$. Firstly, we define obediently a bid valuation $\bar{C}_{t}^{\alpha, n}(B)$ corresponding to our new valuation as follows:

$$
\begin{aligned}
& \operatorname{esssup}_{\vartheta \in \bar{\Theta}_{0}^{(n)}} E\left[-\left(1-\frac{\alpha}{n} G_{t, T}(\vartheta)\right)^{n+1} \mid \mathcal{F}_{t}\right] \\
& =\operatorname{esssup}_{\vartheta \in \bar{\Theta}_{B}^{(n)}} E\left[-\left(1-\frac{\alpha}{n} \bar{C}_{t}^{\alpha, n}(B)\right)^{-n}\left(1-\frac{\alpha}{n} G_{t, T}(\vartheta)\right)^{n+1}\left(1-\frac{\alpha}{n} B\right)^{n} \mid \mathcal{F}_{t}\right],
\end{aligned}
$$

where $\bar{\Theta}_{0}^{(n)}$ and $\bar{\Theta}_{B}^{(n)}$ are suitable spaces of $\mathbf{R}^{d}$-valued predictable processes. However, $\bar{C}_{t}^{\alpha, n}(B)$ is not convenient, because if we denote

$$
\frac{d \bar{P}^{n, B}}{d P}:=\bar{C}^{n, B}\left(1-\frac{\alpha}{n} B\right)^{n},
$$

where $\bar{C}^{n, B}$ is a positive constant, then $\bar{P}^{n, B}$ is not equivalent to $P$ in general. Note that we should set $\bar{\Theta}_{B}^{(n)}=\Theta^{n+1}\left(\bar{P}^{n, B}\right)$ for (6.1). Thus, we suggest, in this section, another definition of a bid valuation, denoted by $\check{C}_{t}^{\alpha, n}(B)$, for $C_{t}^{\alpha, n}(B)$ by an approximate way as follows:

$$
\begin{aligned}
& \operatorname{esssup}_{\vartheta \in \check{\Theta}_{0}^{(n)}} E\left[-\left(1-\frac{\alpha}{n} G_{t, T}(\vartheta)\right)^{n+1} \mid \mathcal{F}_{t}\right] \\
& =\operatorname{esssup}_{\vartheta \in \check{\Theta}_{B}^{(n)}} E\left[-\left(1+\frac{\alpha}{n} \check{C}_{t}^{\alpha, n}(B)\right)^{n}\left(1-\frac{\alpha}{n} G_{t, T}(\vartheta)\right)^{n+1}\left(1+\frac{\alpha}{n} B\right)^{-n} \mid \mathcal{F}_{t}\right],
\end{aligned}
$$


where $\check{\Theta}_{B}^{(n)}:=\Theta^{n+1}\left(\check{P}^{n, B}\right)$ and $\check{P}^{n, B}$ is defined as

$$
\frac{d \check{P}^{n, B}}{d P}:=\check{C}^{n, B}\left(1+\frac{\alpha}{n} B\right)^{-n} .
$$

Note that $\check{C}^{n, B}$ is a positive constant. By the definition of $\check{P}^{n, B}$, there exists the $1+\frac{1}{n}$-optimal martingale measure $\check{Q}^{(n), B}$ with respect to $\check{P}^{n, B}$ in $\mathcal{M}_{1+\frac{1}{n}}^{e}\left(\check{P}^{n, B}\right)$, and its density process $\check{Z}^{(n), B}$ with respect to $\check{P}^{n, B}$ satisfies $\mathcal{R}_{1+\frac{1}{n}}\left(\check{P}^{n, B}\right)$. Moreover, if we denote

$\check{V}_{t}^{\alpha, n, B}\left(x_{t}\right):=\operatorname{esssup}_{\vartheta \in \check{\Theta}_{B}^{(n)}} E\left[-\left(1+\frac{\alpha}{n} x_{t}\right)^{n}\left(1-\frac{\alpha}{n} G_{t, T}(\vartheta)\right)^{n+1}\left(1+\frac{\alpha}{n} B\right)^{-n} \mid \mathcal{F}_{t}\right]$.

then, we have, by the definition of $\check{C}_{t}^{\alpha, n}(B)$,

$$
\check{V}_{t}^{\alpha, n, 0}(0)=\check{V}_{t}^{\alpha, n, B}\left(\check{C}_{t}^{\alpha, n}(B)\right) .
$$

This implies that

$$
\begin{aligned}
& \frac{\check{V}_{t}^{\alpha, n, B}(0)}{\check{V}_{t}^{\alpha, n, 0}(0)} \\
& =\frac{\check{V}_{t}^{\alpha, n, B}(0)}{\check{V}_{t}^{\alpha, n, B}\left(\check{C}_{t}^{\alpha, n}(B)\right)} \\
& =\frac{\operatorname{esssup}_{\vartheta \in \check{\Theta}_{B}^{(n)}} E\left[-\left(1-\frac{\alpha}{n} G_{t, T}(\vartheta)\right)^{n+1}\left(1+\frac{\alpha}{n} B\right)^{-n} \mid \mathcal{F}_{t}\right]}{\operatorname{esssup}_{\vartheta \in \check{\Theta}_{B}^{(n)}} E\left[-\left(1+\frac{\alpha}{n} \check{C}_{t}^{\alpha, n}(B)\right)^{n}\left(1-\frac{\alpha}{n} G_{t, T}(\vartheta)\right)^{n+1}\left(1+\frac{\alpha}{n} B\right)^{-n} \mid \mathcal{F}_{t}\right]} \\
& =\frac{1}{\left(1+\frac{\alpha}{n} \check{C}_{t}^{\alpha, n}(B)\right)^{n} .}
\end{aligned}
$$

Hence, $\check{C}_{t}^{\alpha, n}(B)$ is represented as

$$
\check{C}_{t}^{\alpha, n}(B)=\frac{n}{\alpha}\left\{\left(\frac{\check{V}_{t}^{\alpha, n, B}(0)}{\check{V}_{t}^{\alpha, n, 0}(0)}\right)^{-\frac{1}{n}}-1\right\}
$$

Just as in the ask valuation, we give a duality relationship with respect to $\check{C}_{t}^{\alpha, n}(B)$.

Theorem 6.1 We have the following duality relationship with respect to $\check{C}_{t}^{\alpha, n}(B)$ :

$$
\begin{gathered}
\operatorname{esssup}_{\vartheta \in \check{\Theta}_{B}^{(n)}} E\left[-\left(1-\frac{\alpha}{n} G_{t, T}(\vartheta)\right)^{n+1}\left(1+\frac{\alpha}{n} B\right)^{-n} \mid \mathcal{F}_{t}\right] \\
=-\left\{\operatorname{essinf}_{Q \in \mathcal{M}_{1+\frac{1}{n}}^{e}\left(\check{P}^{n, B}\right)} E_{Q}\left[\left(Z_{t, T}^{Q}\right)^{\frac{1}{n}}\left(1+\frac{\alpha}{n} B\right) \mid \mathcal{F}_{t}\right]\right\}^{-n} .
\end{gathered}
$$


Proof. We denote

$$
\check{Z}_{t, T}^{n, B}:=\frac{\check{Z}_{T}^{n, B}}{\check{Z}_{t}^{n, B}}=\check{C}_{t}^{n, B}\left(1+\frac{\alpha}{n} B\right)^{-n} \quad \text { and } \quad \check{Z}_{t}^{n, B}:=E\left[\frac{d \check{P}^{n, B}}{d P} \mid \mathcal{F}_{t}\right],
$$

where $\check{C}_{t}^{n, B}$ is an $\mathcal{F}_{t}$-measurable positive random variable. Moreover, we denote $\check{Z}_{t, T}^{(n), B}:=\frac{\check{Z}_{T}^{(n), B}}{\check{Z}_{t}^{(n), B}}=\check{C}_{t}^{(n), B}\left(1+\frac{{ }^{t} \check{f}_{T}^{(n), B}}{n}\right)^{n}$ and $\check{Z}_{t}^{(n), B}:=E_{\check{P}^{n, B}}\left[\frac{d \check{Q}^{(n), B}}{d \check{P}^{n, B}} \mid \mathcal{F}_{t}\right]$, where $\check{C}_{t}^{(n), B}$ is an $\mathcal{F}_{t}$-measurable positive random variable, and ${ }^{t} \check{f}_{T}^{(n), B} \in$ ${ }^{t} \mathcal{K}_{n+1}\left(\check{P}^{n, B}\right)$. In particular, $-{ }^{t} \check{f}_{T}^{(n), B} / n$ is the projection of "1" onto ${ }^{t} \mathcal{K}_{n+1}\left(\check{P}^{n, B}\right)$ in $\mathcal{L}^{n+1}\left(\check{P}^{n, B}\right)$. Now, we compute the left hand side of (6.2).

$$
\begin{aligned}
\text { LHS of (6.2) } & =-\frac{1}{\check{C}_{t}^{n, B}} \operatorname{essinf}_{\vartheta \in \check{\Theta}_{B}^{(n)}} E_{\check{P}^{n, B}}\left[\left(1-\frac{\alpha}{n} G_{t, T}(\vartheta)\right)^{n+1} \mid \mathcal{F}_{t}\right] \\
& =-\frac{1}{\check{C}_{t}^{n, B}} E_{\check{P}^{n, B}}\left[\left(1+\frac{{ }^{t} \check{f}_{T}^{(n), B}}{n}\right)^{n+1} \mid \mathcal{F}_{t}\right] \\
& =-\frac{1}{\check{C}_{t}^{n, B}} \frac{1}{\check{C}_{t}^{(n), B}} E_{\check{Q}^{(n), B}}\left[\left(1+\frac{{ }^{t} \check{f}_{T}^{(n), B}}{n}\right) \mid \mathcal{F}_{t}\right] \\
& =-\frac{1}{\check{C}_{t}^{n, B}} \frac{1}{\check{C}_{t}^{(n), B}} .
\end{aligned}
$$

On the other hand, we have

RHS of $(6.2)$

$$
\begin{aligned}
& =-\left\{\operatorname{essinf}_{Q \in \mathcal{M}_{1+\frac{1}{n}}^{e}}\left(\check{P}^{n, B}\right) E\left[\left(\check{Z}_{t, T}^{Q, n, B} \check{Z}_{t, T}^{n, B}\right)^{1+\frac{1}{n}}\left(\check{Z}_{t, T}^{n, B}\right)^{-\frac{1}{n}}\left(\check{C}_{t}^{n, B}\right)^{\frac{1}{n}} \mid \mathcal{F}_{t}\right]\right\}^{-n} \\
& =-\frac{1}{\check{C}_{t}^{n, B}}\left\{\operatorname{essinf}_{Q \in \mathcal{M}_{1+\frac{1}{n}}^{e}\left(\check{P}^{n, B}\right)} E_{\check{P}^{n, B}}\left[\left(\check{Z}_{t, T}^{Q, n, B}\right)^{1+\frac{1}{n}} \mid \mathcal{F}_{t}\right]\right\}^{-n} \\
& =-\frac{1}{\check{C}_{t}^{n, B}}\left\{E_{\check{Q}^{(n), B}}\left[\left(\check{Z}_{t, T}^{(n), B}\right)^{\frac{1}{n}} \mid \mathcal{F}_{t}\right]\right\}^{-n} \\
& =-\frac{1}{\check{C}_{t}^{n, B}} \frac{1}{\check{C}_{t}^{(n), B}}
\end{aligned}
$$

where

$$
\check{Z}_{t, T}^{Q, n, B}:=\frac{\check{Z}_{T}^{Q, n, B}}{\check{Z}_{t}^{Q, n, B}} \quad \text { and } \quad \check{Z}_{t}^{Q, n, B}:=E_{\check{P}^{n, B}}\left[\frac{d Q}{d \check{P}^{n, B}} \mid \mathcal{F}_{t}\right]
$$

This completes the proof of Theorem 6.1.

By using Theorem 6.1, we obtain another representation of $\check{C}_{t}^{\alpha, n}(B)$. 
Corollary 6.2 Denote

$$
\widetilde{\tilde{V}}_{t}^{\alpha, n, B}:=\operatorname{essinf}_{Q \in \mathcal{M}_{1+\frac{1}{n}}^{e}}\left(\check{P}^{n, B}\right) E_{Q}\left[\left(Z_{t, T}^{Q}\right)^{\frac{1}{n}}\left(1+\frac{\alpha}{n} B\right) \mid \mathcal{F}_{t}\right] .
$$

Then, we have

$$
\check{V}_{t}^{\alpha, n, B}(0)=-\left(\widetilde{\widetilde{V}}_{t}^{\alpha, n, B}\right)^{-n}
$$

and

$$
\check{C}_{t}^{\alpha, n}(B)=\frac{n}{\alpha}\left\{\frac{\widetilde{\widetilde{V}}_{t}^{\alpha, n, B}}{\widetilde{\widetilde{V}}_{t}^{\alpha, n, 0}-1}\right\} .
$$

As in Sections 4 and 5, we have to investigate whether or not $\check{C}_{t}^{\alpha, n}(B)$ satisfies Proposition 4.3 approximately and converges to the EUIV. Since $\bar{C}_{t}^{\alpha, n}(B)$ defined in (6.1) is equivalent to $-C_{t}^{\alpha, n}(-B)$ as long as $B$ is bounded and $n$ is a sufficient large, we can prove easily that $\bar{C}_{t}^{\alpha, n}(B)$ approximately satisfies (1), (2) and (4) of Proposition 4.3, and the reverse inequality of (3), that is, we can say that $\bar{C}_{t}^{\alpha, n}(B)$ is an approximate concave monetary utility functional. Thus, we have only to study the relationship between $\check{C}_{t}^{\alpha, n}(B)$ and $\bar{C}_{t}^{\alpha, n}(B)$ in order to confirm that $\check{C}_{t}^{\alpha, n}(B)$ is meaningful as a bid valuation of our new approximate approach to the EUIV.

Theorem 6.3 For $B \in \mathcal{L}_{+}^{\infty}(P)$ and any sufficient large $n$, there exists a constant $C>0$ depending on $\|B\|_{\infty}$ such that

$$
\sup _{0 \leq t \leq T}\left|\check{C}_{t}^{\alpha, n}(B)-\bar{C}_{t}^{\alpha, n}(B)\right| \leq C \frac{\alpha}{n} .
$$

Proof. Remark that, since $B$ is bounded and $n$ is a sufficient large, $\bar{P}^{n, B}$ is equivalent to $P$ and $\Theta_{B}^{(n)}=\check{\Theta}_{B}^{(n)}=\bar{\Theta}_{B}^{(n)}$ holds. Now, we define $\check{C}_{t}^{\alpha, n}(B)$ as an adapted process satisfying

$$
\begin{aligned}
& \operatorname{esssup}_{\vartheta \in \Theta_{B}^{(n)}} E\left[-\left(1-\frac{\alpha}{n} G_{t, T}(\vartheta)\right)^{n+1} \mid \mathcal{F}_{t}\right] \\
& =\operatorname{esssup}_{\vartheta \in \Theta_{B}^{(n)}} E\left[-\left(1-\frac{\alpha}{n} \check{C}_{t}^{\alpha, n}(B)\right)^{-n}\left(1-\frac{\alpha}{n} G_{t, T}(\vartheta)\right)^{n+1}\left(1+\frac{\alpha}{n} B\right)^{-n} \mid \mathcal{F}_{t}\right] .
\end{aligned}
$$

Then, we have

$$
\check{\check{C}}_{t}^{\alpha, n}(B)=\frac{\check{C}_{t}^{\alpha, n}(B)}{1+\frac{\alpha}{n} \check{C}_{t}^{\alpha, n}(B)}=-\frac{n}{\alpha}\left\{\frac{\widetilde{\widetilde{V}}_{t}^{\alpha, n, 0}}{\widetilde{\widetilde{V}}_{t}^{\alpha, n, B}}-1\right\} .
$$

We prove that there exists a $C>0$ such that $\bar{C}_{t}^{\alpha, n}(B)-\check{C}_{t}^{\alpha, n}(B) \leq C \frac{\alpha}{n}$. Note that we have

$$
\bar{C}_{t}^{\alpha, n}(B)-\check{\check{C}}_{t}^{\alpha, n}(B)=-C_{t}^{\alpha, n}(-B)-\check{\check{C}}_{t}^{\alpha, n}(B)
$$




$$
\begin{aligned}
& =-\frac{n}{\alpha}\left\{\frac{\widetilde{V}_{t}^{\alpha, n, 0}}{\widetilde{V}_{t}^{\alpha, n,-B}}-1\right\}+\frac{n}{\alpha}\left\{\frac{\widetilde{\widetilde{V}}_{t}^{\alpha, n, 0}}{\widetilde{\widetilde{V}}_{t}^{\alpha, n, B}}-1\right\} \\
& =\frac{n}{\alpha} \frac{\widetilde{V}_{t}^{\alpha, n, 0}}{\widetilde{\widetilde{V}}_{t}^{\alpha, n, B}} \frac{\widetilde{V}_{t}^{\alpha, n,-B}-\widetilde{V}_{t}^{\alpha, n, B}}{\widetilde{V}_{t}^{\alpha, n,-B}} .
\end{aligned}
$$

We estimate the right hand side of the above. Firstly, we have

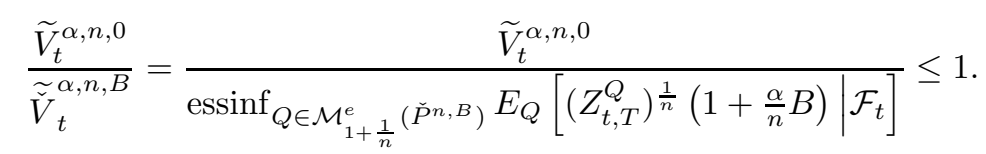

Next, we compute $\widetilde{V}_{t}^{\alpha, n,-B}-\widetilde{\tilde{V}}_{t}^{\alpha, n, B}$ as follows:

$$
\begin{aligned}
& \widetilde{V}_{t}^{\alpha, n,-B}-\widetilde{\widetilde{V}}_{t}^{\alpha, n, B} \\
&=\operatorname{essinf}_{Q \in \mathcal{M}_{1+\frac{1}{n}}^{e}\left(\check{P}^{n, B}\right)} E_{Q}\left[\left(Z_{t, T}^{Q}\right)^{\frac{1}{n}}\left(1-\frac{\alpha}{n} B\right)^{-1} \mid \mathcal{F}_{t}\right] \\
&-\operatorname{essinf}_{Q \in \mathcal{M}_{1+\frac{1}{n}}^{e}}\left(\check{P}^{n, B}\right) E_{Q}\left[\left(Z_{t, T}^{Q}\right)^{\frac{1}{n}}\left(1+\frac{\alpha}{n} B\right) \mid \mathcal{F}_{t}\right] \\
&= \operatorname{essinf}_{Q \in \mathcal{M}_{1+\frac{1}{n}}^{e}\left(\check{P}^{n, B}\right)} E_{Q}\left[\left(Z_{t, T}^{Q}\right)^{\frac{1}{n}}\left(1+\frac{\alpha}{n} B+\left(\frac{\alpha}{n} B\right)^{2}\left(1-\frac{\alpha}{n} B\right)^{-1}\right) \mid \mathcal{F}_{t}\right] \\
&-\operatorname{essinf}_{Q \in \mathcal{M}_{1+\frac{1}{n}}^{e}}\left(\check{P}^{n, B}\right) \\
& \leq E_{Q}\left[\left(Z_{t, T}^{Q}\right)^{\frac{1}{n}}\left(1+\frac{\alpha}{n} B\right) \mid \mathcal{F}_{t}\right] \\
& \leq\left.\left(\frac{\alpha}{n}\|B\|_{\infty}^{Q^{\prime}}\right)^{\frac{1}{n}}\left(1+\frac{\alpha}{n} B+\left(\frac{\alpha}{n} B\right)^{2}\left(1-\frac{\alpha}{n} B\right)^{-1}-\left(1+\frac{\alpha}{n} B\right)\right) \mid \mathcal{F}_{t}\right]
\end{aligned}
$$

where

$\operatorname{essinf}_{Q \in \mathcal{M}_{1+\frac{1}{n}}^{e}\left(\check{P}^{n, B}\right)} E_{Q}\left[\left(Z_{t, T}^{Q}\right)^{\frac{1}{n}}\left(1+\frac{\alpha}{n} B\right) \mid \mathcal{F}_{t}\right]=E_{Q^{\prime}}\left[\left(Z_{t, T}^{Q^{\prime}}\right)^{\frac{1}{n}}\left(1+\frac{\alpha}{n} B\right) \mid \mathcal{F}_{t}\right]$.

Remark that $\mathcal{M}_{1+\frac{1}{n}}^{e}\left(P^{n, B}\right)=\mathcal{M}_{1+\frac{1}{n}}^{e}\left(\check{P}^{n, B}\right)$. Moreover, we have

$$
\begin{aligned}
\tilde{V}_{t}^{\alpha, n,-B} & =\operatorname{essinf}_{Q \in \mathcal{M}_{1+\frac{1}{n}}^{e}}\left(\check{P}^{n, B}\right) \\
& \geq E_{Q}\left[\left(Z_{t, T}^{Q}\right)^{\frac{1}{n}}\left(1-\frac{\alpha}{n} B\right)^{-1} \mid \mathcal{F}_{t}\right] \\
& =E_{Q^{\prime}}\left[\left(Z_{t, T}^{Q^{\prime}}\right)^{\frac{1}{n}}\left(1+\frac{\alpha}{n} B\right) \mid \mathcal{F}_{t}^{e}\right] \\
& \geq E_{Q^{\prime}}\left[\left(Z_{t, T}^{Q^{\prime}}\right)^{\frac{1}{n}} \mid \mathcal{F}_{t}\right] .
\end{aligned}
$$

As a result, we obtain

$$
\frac{\widetilde{V}_{t}^{\alpha, n,-B}-\widetilde{\widetilde{V}}_{t}^{\alpha, n, B}}{\widetilde{V}_{t}^{\alpha, n,-B}} \leq\left(\frac{\alpha}{n}\|B\|_{\infty}\right)^{2}\left(1-\frac{\alpha}{n}\|B\|_{\infty}\right)^{-1} .
$$


Hence, we can conclude that there exists a $C>0$ such that

$$
\bar{C}_{t}^{\alpha, n}(B)-\check{C}_{t}^{\alpha, n}(B) \leq \frac{n}{\alpha}\left(\frac{\alpha}{n}\|B\|_{\infty}\right)^{2}\left(1-\frac{\alpha}{n}\|B\|_{\infty}\right)^{-1} \leq C \frac{\alpha}{n} .
$$

On the other hand, since $\widetilde{V}_{t}^{\alpha, n,-B}-\widetilde{\breve{V}}_{t}^{\alpha, n, B} \geq 0$, we obtain $\bar{C}_{t}^{\alpha, n}(B)-$ $\check{C}_{t}^{\alpha, n}(B) \geq 0$. Moreover, since $\check{C}_{t}^{\alpha, n}(B) \leq\|B\|_{\infty}$, there exists a $C>0$ such that

$$
0 \leq \check{C}_{t}^{\alpha, n}(B)-\check{C}_{t}^{\alpha, n}(B) \leq \check{C}_{t}^{\alpha, n}(B)-\frac{\check{C}_{t}^{\alpha, n}(B)}{1+\frac{\alpha}{n} \check{C}_{t}^{\alpha, n}(B)} \leq C \frac{\alpha}{n} .
$$

Thus, Theorem 6.3 follows.

Consequently, the bid valuation $\check{C}_{t}^{\alpha, n}(B)$ satisfies approximately the same basic properties as a concave monetary utility functional, and converges to the bid valuation of the EUIV in probability.

\section{Concluding Remarks}

The results of Sections 4, 5 and 6 mean that, under some assumptions related to the $1+\frac{1}{n}$-optimal martingale measure, our new valuations $C_{t}^{\alpha, n}(B)$ and $\check{C}_{t}^{\alpha, n}(B)$ are available as approximate approaches to the EUIV for ask and bid, respectively. In other words, we succeed in relaxing the condition (1.2) to (1.3) for a sufficient large $n$ by using a kind of power functions. In addition, Section 3 shows that there exist many important examples satisfying the all standing assumptions.

On the other hand, in order that we calculate our valuation $C_{t}^{\alpha, n}(B)$ concretely, we need to obtain the density of $P^{n, B}$ and the projection of " 1 " onto ${ }^{t} \mathcal{K}_{n+1}\left(P^{n, B}\right)$. These are big difficulties for us to realize our new valuation. However, there exist several cases where we can compute concretely the density of the $p$-optimal martingale measure, for example, see Hobson (2004).

Finally, we eliminate some future problems. We have not researched for the dynamics of our new valuation in this paper. Hence, very little is known about properties of our valuation as processes. For example, the time-consistent or the locally Lipschitz continuity in $\alpha$ (see Section 5 of MS). Furthermore, we are interested in the asymptotic behavior of $C_{t}^{\alpha, n}(B)$ as $\alpha$ tends to 0 or $\infty$. For example, MS have proved that the EUIV converges to the conditional expectation under the minimal entropy martingale measure as $\alpha$ tends to 0 , and to the superreplication price process as $\alpha$ tends to $\infty$.

Acknowledgments The author would like to express his gratitude to Martin Schweizer and Shigeo Kusuoka for their much valuable advice. The financial support of the author has been partially granted by Grant-in-Aid for Young Scientists (B) No.16740062 from the Ministry of Education, Culture, Sports, Science and Technology of Japan. 


\section{References}

[1] Becherer, D. (2004) Utility-indifference hedging and valuation via reaction-diffusion systems. Proc. Royal Society London A 460, 27-51.

[2] Cheridito, P., Delbaen F. and Kupper, M. (2004) Coherent and convex monetary risk measures for bounded cádlág processes. Stoch. Proc. Appl. $112,1-22$.

[3] Choulli, T., Krawczyk, L. And Stricker, C. (1998) $\mathcal{E}$-martingales and their applications in mathematical finance. Ann. Prob. 26, 853-876.

[4] Choulli, T., Krawczyk, L. and Stricker, C. (1999) On Fefferman and Burkholder-Davis-Gundy inequalities for $\mathcal{E}$-martingales. Prob. Th. Rel. Field 113, 571-597.

[5] Dellacherie, C. And Meyer, P.A. (1982) Probabilities and Potential B., North-Holland, Amsterdam.

[6] Föllmer, H. ANd Schweizer, M. (1991) Hedging of contingent claims under incomplete information. In Applied stochastic analysis (London, 1989), (Stochastics Monogr. 5) Gordon and Breach, pp.389-414.

[7] Frittelli, M. (2000) Introduction to a theory of value coherent with the no-arbitrage principle. Fin. Stoch. 4, 275-297.

[8] Grandits, P. and Krawczyk, L. (1998) Closedness of some spaces of stochastic integrals. In Séminaire de Probabilités, XXXII (Lecture Notes in Math. 1686), Springer, pp.73-85.

[9] Grandits, P. and Rheinländer, T. (2002) On the minimal entropy martingale measure. Ann. Prob. 30, 1003-1038.

[10] Hobson, D. (2004) Stochastic volatility models, correlation, and the $q$ optimal measure. Math. Fin. 14, 537-556.

[11] Mania, M. and Schweizer, M. (2005) Dynamic exponential utility indifference valuation. to appear in Ann. Appl. Probab..

[12] Musiela, M. and Zariphopoulou, T. (2004a) An example of indifference pricing under exponential preferences. Fin. Stoch. 8, 229-239.

[13] Musiela, M. and Zariphopoulou, T. (2004b) A valuation algorithm for indifference prices in incomplete markets. Fin. Stoch. 8, 399-414.

[14] Protter, P. (1990) Stochastic Integration and Differential Equations. A new approach, Springer-Verlag, Berlin.

[15] Rouge, R. and El Karoui, N. (2000) Pricing via utility maximization and entropy. Math. Fin. 10, 259-276.

[16] Young, V. (2004) Pricing in an incomplete market with an affine term structure. Math. Fin. 14, 359-381. 